Document downloaded from:

http://hdl.handle.net/10251/166915

This paper must be cited as:

Orlando, JI.; Fu, H.; Breda, JB.; Van Keer, K.; Bathula, DR.; Diaz-Pinto, A.; Fang, R.... (2020). REFUGE Challenge: A unified framework for evaluating automated methods for glaucoma assessment from fundus photographs. Medical Image Analysis. 59:1-21. https://doi.org/10.1016/j.media.2019.101570

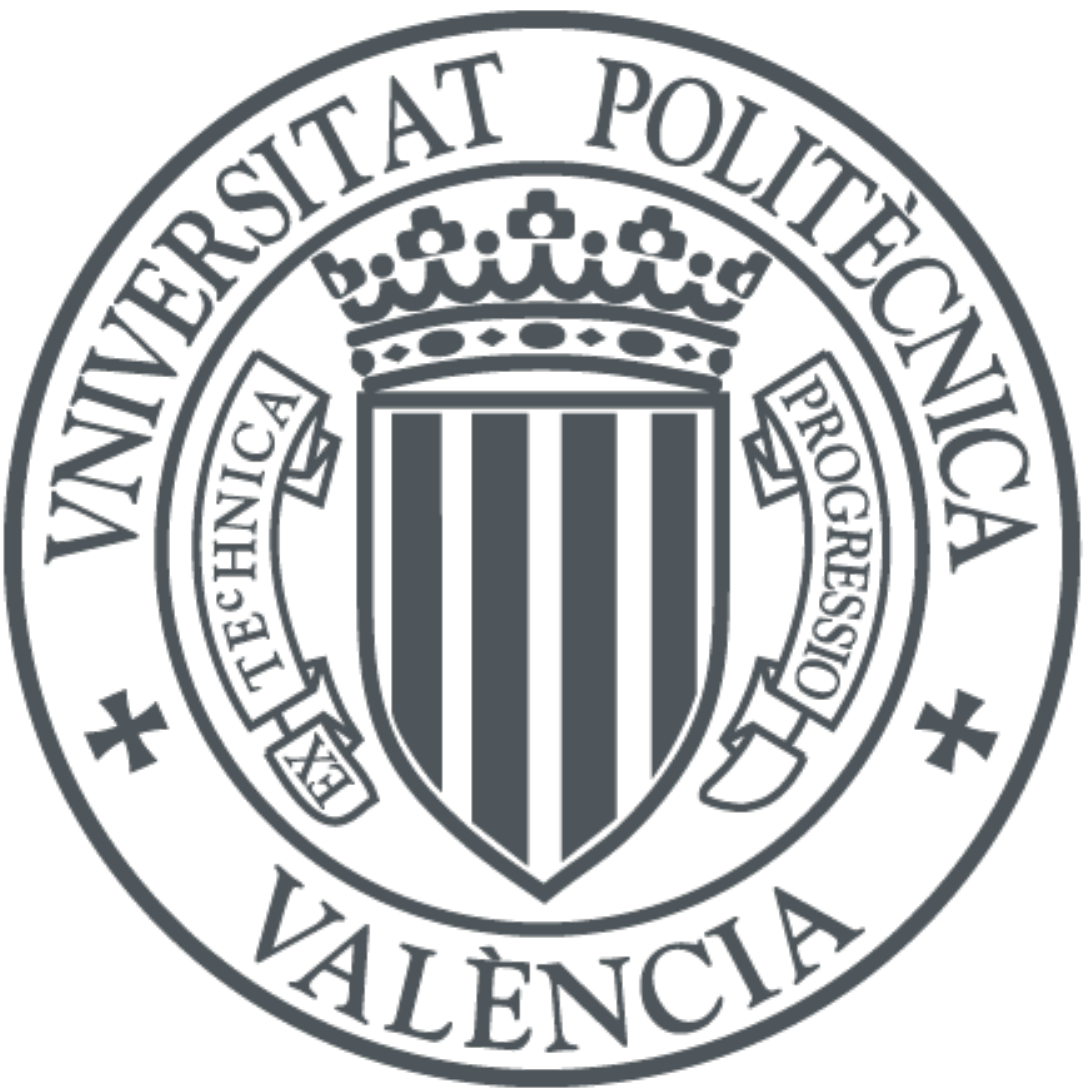

The final publication is available at

https://doi.org/10.1016/j.media.2019.101570

Copyright Elsevier

Additional Information 


\title{
REFUGE Challenge: A Unified Framework for Evaluating Automated Methods for Glaucoma Assessment from Fundus Photographs
}

\author{
José Ignacio Orlando, Huazhu Fu, João Barbossa Breda, Karel van Keer, Deepti R. Bathula, Andrés Yesid
} Diaz-Pinto, Ruogu Fang, Pheng-Ann Heng, Jeyoung Kim, JoonHo Lee, Joonseok Lee, Xiaoxiao Li, Peng Liu, Shuai Lu, Balamurali Murugesan, Valery Naranjo, Sai Samarth R. Phaye, Sharath M. Shankaranarayana, Apoorva Sikka, Jaemin Son, Anton van den Hengel, Shujun Wang, Junyan Wu, Zifeng Wu, Guanghui Xu, Yongli Xu, Pengshuai Yin, Xiulan Zhang, Fei Li, Hrvoje Bogunović, and Yanwu Xu

\begin{abstract}
Glaucoma is one of the leading causes of irreversible
\end{abstract}
Manuscript received March XX, 2019; revised XXXXX, 2019. (Corresponding author: Hrvoje Bogunović.)

Yanwu Xu, José Ignacio Orlando, Hrvoje Bogunović, Huazhu Fu, Xiulan Zhang and Fei Li organized the challenge. João Barbossa Breda and Karel van Keer were clinical partners. Participants who provided short descriptions of their methods for the appendix are listed alphabetically.

J. I. Orlando (jose.orlando@meduniwien.ac.at) and H. Bogunović (hrvoje.bogunovic@meduniwien.ac.at) were with the Christian Doppler Laboratory for Ophthalmic Image Analysis (OPTIMA), Vienna Reading Center (VRC), Department of Ophthalmology and Optometry, Medical University of Vienna, 1200 Vienna, Austria.

Huazhu Fu was with Inception Institute of Artificial Intelligence, Abu Dhabi, United Arab Emirates (hzfu@ieee.org)

João Barbossa Breda (joao.breda@med.up.pt) was with the Surgery and Physiology Department, Ophthalmology Unit, Faculty of Medicine of the University of Porto, Porto, Portugal, and Research Group Ophthalmology, KU Leuven, Leuven, Belgium

Karel Van Keer (karel.vankeer@uzleuven.be) was with the Research Group Ophthalmology, KU Leuven, Leuven, Belgium.

Apoorva Sikka (apoorva.sikka@iitrpr.ac.in), Sai Samarth R. Phaye and Deepti R. Bathula were part of the NightOwl team and the Indian Institute of Technology Ropar, Punjab, India.

Pengshuai Yin (pshuai.yin@gmail.com) and Guanghui Xu were part of the Masker team and South China University of Technology, Guangzhou, China.

Andrés Yesid Diaz-Pinto (andiapin@i3b.upv.es) and Valery Naranjo were part of the Cvblab team and Instituto de Investigación e Innovación en Bioingeniería, I3B, Universitat Politècnica de València, 46022 Valencia, Spain.

Sharath M Shankaranarayana (ee15s050@ee.iitm.ac.in) and Balamurali Murugesan were part of the WinterFell team. S. M. Shankaranarayana was with the Department of Electrical Engineering, IIT-Madras, India. B. Murugesan was with Healthcare Technology Innovation Centre, IIT-Madras, India.

Peng Liu and Ruogu Fang were part of the SMILEDeepDR and J. Crayton Pruitt Family Dept. of Biomedical Engineering, University of Florida, USA.

Zifeng Wu and Anton van den Hengel were part of the AIML team and Australian Institute for Machine Learning, Australia.

Junyan $\mathrm{Wu}$ and Xiaoxiao $\mathrm{Li}$ were part of the Mammoth team. Junyan Wu was with Cleerly Inc. New York City, NY USA. Xiaoxiao Li was with Yale University, New Haven, CT USA.

Shujun Wang (sjwang@cse.cuhk.edu.hk) and Pheng-Ann Heng (pheng@cse.cuhk.edu.hk) were part of the CUHKMED team and the Chinese University of Hong Kong, Hong Kong.

Jaemin Son and Jeyoung Kim were part of the VRT team. J. Son was with VUNO Inc., Seoul, Korea (woalsdnd@ vuno.co). J. Kim was with Gachon University, Gyeonggi-do, Korea.

Yongly Xu and Shuai Lu were part of the BUCT team and with Faculty of Science, Beijing University of Chemical Technology, Beijin, China.

Joonseok Lee (js1985.lee@samsung.com) and JoonHo Lee (joonholee@samsung.com) were part of the SDSAIRC team, and with Samsung SDS AI Research Center, Seoul, Korea.

Yanwu Xu was with Artificial Intelligence Innovation Business, Baidu Inc., China and Cixi Institute of BioMedical Engineering, Chinese Academy of Sciences, China. but preventable blindness in working age populations. Color fundus photography (CFP) is the most cost-effective imaging modality to screen for retinal disorders. However, its application to glaucoma has been limited to the computation of a few related biomarkers such as the vertical cup-to-disc ratio. Deep learning approaches, although widely applied for medical image analysis, have not been extensively used for glaucoma assessment due to the limited size of the available data sets. Furthermore, the lack of a standardize benchmark strategy makes difficult to compare existing methods in a uniform way. In order to overcome these issues we set up the Retinal Fundus Glaucoma Challenge, REFUGE (https://refuge.grand-challenge.org), held in conjunction with MICCAI 2018. The challenge consisted of two primary tasks, namely optic disc/cup segmentation and glaucoma classification. As part of REFUGE, we have publicly released a data set of 1200 fundus images with ground truth segmentations and clinical glaucoma labels, currently the largest existing one. We have also built an evaluation framework to ease and ensure fairness in the comparison of different models, encouraging the development of novel techniques in the field. 12 teams qualified and participated in the online challenge. This paper summarizes their methods and analyzes their corresponding results. In particular, we observed that two of the top-ranked teams outperformed two human experts in the glaucoma classification task. Furthermore, the segmentation results were in general consistent with the ground truth annotations, with complementary outcomes that can be further exploited by ensembling the results.

Index Terms_retinal imaging; glaucoma; fundus photography; grand challenge

\section{INTRODUCTION}

$\mathbf{G}$ LAUCOMA is a chronic neuro-degenerative condition that is one of the leading causes of irreversible but preventable blindness in the world [1]. In 2013, 64.3 million people aged 40-80 years were estimated to suffer from glaucoma, while this number is expected to increase to 76 million by 2020 and 111.8 million by 2040 [1]. In its many variants, glaucoma is characterized by the damage of the optic nerve head $(\mathrm{ONH})$, typically caused by a high intra-ocular pressure (IOP). IOP is increased as a consequence of abnormal accumulation of aqueous humor in the eye, induced by pathological defects in the eye's drainage system. When the anterior segment is saturated with this fluid, the IOP progressively elevates, compressing the vitreous to the retina. If this remains uncontrolled, it can produce damage in the nerve fiber layer, the vasculature 
and the $\mathrm{ONH}$, leading to a progressive and irreversible vision loss that can ultimately result in blindness. As this process occurs asymptomatically, glaucoma is frequently referred as the "silent thief of sight" [2]: patients are not aware of the progressing disease until the vision is irreversibly lost.

Life-long pharmacological treatments based on the regular administration of eye drops are usually prescribed to control the IOP and to temper further damage in the retina. Alternatively, laser procedures and other surgeries can be performed to increase the drainage. In any case, early detection is essential to prevent vision loss [2]. Unfortunately, at least half of patients with glaucoma currently remain undiagnosed [3]. Being glaucoma a chronic condition, one of the major challenges is to be able to detect this large number of undiagnosed patients [3]. Generalized screening programs have not been employed because of the large amount of false positives these can generate. These misdiagnoses cannot be absorbed by current healthcare infrastructures and would have an unnecessary negative impact on the patient's quality of life, until it would be recognized that no glaucomatous neuropathy existed [2].

Color fundus photography (CFP, Fig. 1) is currently the most economical, non-invasive imaging modality for inspecting the retina [4], [5]. Its widespread availability makes it ideal for assessing several ophthalmic diseases such as agerelated macular degeneration (AMD) [6], diabetic retinopathy (DR) [7] and glaucoma [8]. Screening campaigns can be aided by the incorporation of computer-assisted tools for imagebased diagnosis. As these initiatives require to manually grade a large number of cases in a short period of time, automated tools can help clinians by providing them with quantitative and/or qualitative feedback (e.g. disease likelihood, segmentations of relevant lesions and pathological structures, etc). These approaches have already been successfully applied for detecting DR, in a FDA-approved autonomous diagnostic system, a first of its kind [9]. However, the broad application of similar methods for glaucoma detection is still pending. This is partially due to the fact that the earlier signs of glaucoma are not so easily recognizable in CFP [10] (Fig. 2). In current best clinical practice, CFPs are complementary to other studies such as IOP measurements, automated perimetry and optical coherence tomography (OCT). This approach is not costeffective to be applied for large scale population screening for glaucoma [2]. Therefore, developing automated tools to better exploit the information in CFP is paramount to reduce this burden and ensure an effective detection of glaucoma suspects.

A significant research effort has been made to introduce automated tools for segmenting the optic disc (OD) and the optic cup (OC) in CFP automatically, or to identify glaucomatous cases based on alternative features [11]-[13]. Nevertheless, these approaches currently cannot be properly compared due to the lack of a unified e valuation $\mathrm{f}$ ramework $\mathrm{t}$ o validate them. Moreover, the absence of large scale public available data sets of labeled glaucomatous images has hampered the rapid deployment of deep learning techniques for glaucoma detection [14]. It has been recently shown that image analysis competitions in general can aid to identify challenging scenarios that need further development [15]. Recent grand challenges such as ROC [16], Kaggle [17] and IDRiD [18],

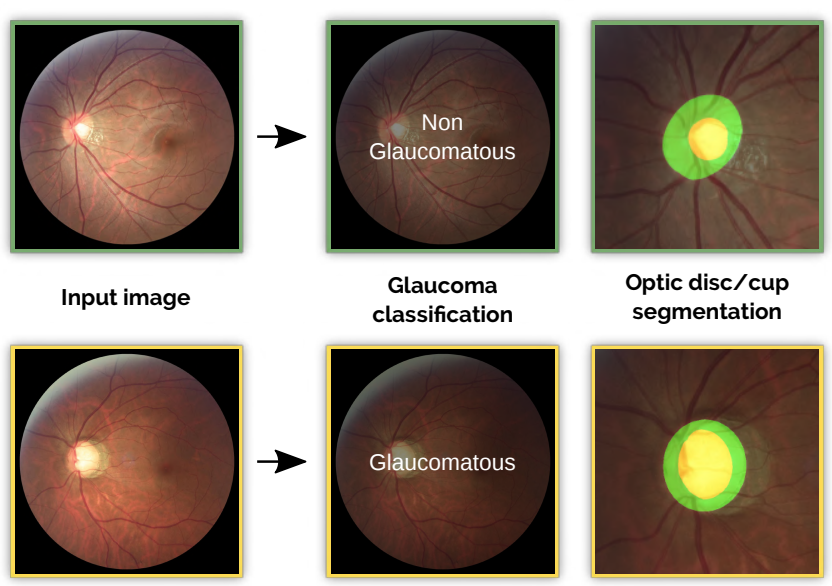

Fig. 1. REFUGE challenge tasks: glaucoma classification and optic disc/cup segmentation from color fundus photographs.

on the other hand, have shown to be useful to address both inconveniences in DR [5], favoring the deployment of these tools into the daily clinical practice [9]. Unfortunately, similar initiatives have not been introduced for glaucoma detection and/or assessment yet.

In an effort to overcome these limitations, we introduced the Retinal Fundus Glaucoma Challenge (REFUGE), a competition that was held as part of the Ophthalmic Medical Image Analysis (OMIA) workshop at MICCAI 2018. The key contributions of the challenge were: (i) the release of a large database (approximately two times bigger than the largest available so far) of 1200 CFP with reliable reference standard annotations for glaucoma identification, optic disc/cup (OD/OC) segmentation and fovea localization; and (ii) the constitution of a unified evaluation framework that enables a standardized fair protocol to compare different algorithms. To the best of our knowledge, REFUGE is the first initiative to provide these key tools at such a large scale. REFUGE participants were invited to use the data set to train and evaluate their algorithms for glaucoma classification and OD/OC segmentation. Their results were quantitatively evaluated using our uniform protocol, to ensure a fair comparison.

In this paper, we analyze the outcomes and the methodological contributions of REFUGE. We present and describe the challenge, reporting the performance of the best algorithms evaluated in the competition and identifying successful common practices for solving the proposed tasks. The results are contrasted with the outcomes of two glaucoma experts to study their performance with respect to independent human observers. Finally, we take advantage of all these empirical evidence to discuss the clinical implications of the results and to propose further improvements to this evaluation framework. In line with the recommendations suggested in [19], REFUGE data and evaluation remain open to encourage further developments and ensure a proper and fair comparison of those new proposals. 

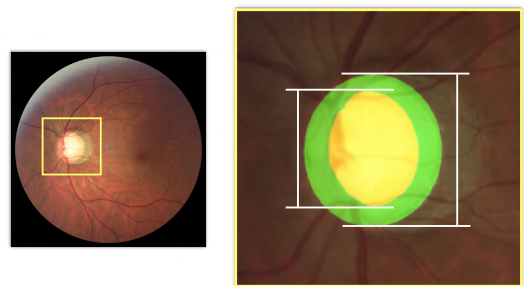

(a) Cupping in the optic nerve head (ONH)

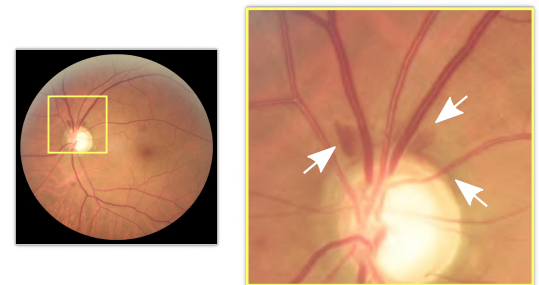

(b) Peripapillary hemorrhages
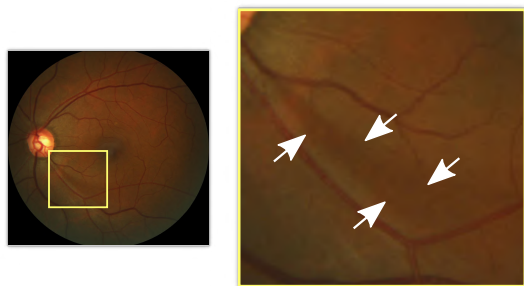

(c) Retinal nerve fiber layer (RNFL) defects

Fig. 2. Pathological changes typical from glaucoma, as observed through fundus photography. (a) Neuroretinal rim thinning due to cupping in the optic nerve head $(\mathrm{ONH})$. White lines indicate the vertical diameter of the optic disc (green) and the optic cup (yellow). (b) Peripapillary hemorrhages, observed as flame-shaped bleedings in the vicinity of the $\mathrm{ONH}$. (c) Retinal nerve fiber layer defects are observed as subtle striations spanning from the optic disc border

\section{Automated glaucoma assessment: STATE-OF-THE-ART AND CURRENT EVALUATION PROTOCOLS}

Early attempts for glaucoma classification and OD/OC segmentation were mostly based on hand-crafted methods using a combination of feature extraction techniques and supervised or unsupervised machine learning classifiers [11] $-[13]$. However, their accuracy was limited due to the application of manually designed features, which are unable to comprehensively characterize the large variability of disease appearance. Deep learning techniques, on the contrary, automatically learn these characteristics by exploiting the implicit information of large training sets of annotated images [20]. In this section we briefly analyze the state-of-the-art techniques for glaucoma classification and OD/OC segmentation and their main evaluation issues. The interested reader could refer to [11] $-[13]$ for a comprehensive analysis of the previous non-deep learning based approaches.

\section{A. Glaucoma classification}

Glaucoma classification consists in categorizing an input CFP into glaucomatous or non-glaucomatous, based on its visual characteristics. Table [I summarizes the most recent methods introduced for this task. In general, most of them are based on adaptations of standard deep supervised learning techniques that are customized to deal with small training sets (Section II-C). In [21], [22], [30], authors proposed to use shallow architectures with a limited number of layers. This is useful to prevent overfitting but limits the ability of the networks to learn rare, specific features. Alternatively, other studies [23], [25], [27] used transfer learning methods, based on deeper architectures but pre-trained on non-medical data. In [25], authors fine-tuned a network initialized with weights learned from ImageNet [32] to detect glaucomatous optic neuropathy. Similarly, transfer learning was shown in [31] to outperform networks trained from scratch for glaucoma detection. Both studies applied a massive image data set with more than 14.000 images to fine tune these networks. Other studies such as [23], [27] used deep learning features extracted from the last fully connected layers of pre-trained networks. The classification task was then performed using linear classifiers trained with these features [23], [27]. This allows to use smaller data sets, although at the cost of lower performance.
Another widely used approach is to restrict the area of analysis to the $\mathrm{ONH}$. This regions is the one that is mostly affected by glaucoma, and focusing only there grant a better exploitation of model parameters. This was done by most of the methods in Table [ and resulted in better performance than learning from full size images. However, such a strong restriction in the networks' field of view hampers their ability to learn alternative features from other regions and therefore their utility for biomarker discovery [21].

\section{B. Optic disc/cup segmentation}

Segmenting the OD and the OC from CFPs is a challenging but relevant task that helps to assess glaucomatous damage to the ONH [11]. Automated methods have to be robust against complex pathological changes such as peripapillary atrophies (PPA) or hemorrhages [12], [13] (Fig. 2 (b)). On the other hand, the accurate delineation of the OC is specially difficult due to the high vessel density in the area and the lack of depth information in CFP [43]. Alternative features such as vessels bendings [44] or intensity changes [45] have been studied in the past to approximate the ONH depth. Table II summarizes current deep learning methods for simultaneous $\mathrm{OD} / \mathrm{OC}$ segmentation.

Most of existing methods use a surrogate segmentation/detection approach to first localize the $\mathrm{ONH}$ area and them crop the images around it [33]-[35], [37], [38]. This prevents false positive detections in regions with e.g. severe illumination artifacts and grants a better exploitation of model parameters, as they are only dedicated to characterize the local appeareance of the OD/OC and not to differentiate these structures from other fundus regions. Alternatively, a two-stage approach was followed in [39], using a first neural network to retrieve a coarse segmentation and a second one to refine the results.

Different neural network architectures have been proposed for OD/OC segmentation. In [33], a classification network similar to LeNet [46] was applied at a patch level to classify its central pixel as belonging to the OD, the OC or the background. Using patches as training samples artificially increases the available training data, although at the cost of loosing spatial information. Alternatively, Zilly et al. proposed to overcome the data limitation issue by training a convolutional neural network using an entropy sampling approach instead of gradient descent. Most of the recent methods [35]-[39], 
TABLE I

OVERVIEW OF EXISTING DEEP LEARNING BASED METHODS FOR GLAUCOMA CLASSIFICATION FROM COLOR FUNDUS PHOTOGRAPHS, SORTED BY THEIR YEAR OF PUBLICATION. $n$ INDICATES THE NUMBER OF IMAGES USED FOR TRAINING/TEST. DIFFERENT TRAINING/TEST DATA COMBINATIONS ARE SEPARATED WITH BARS ("l”). AUC: AREA UNDER THE ROC CURVE. SE: SENSITIVITY, TRUE POSITIVE RATE OR RECALL. SP: SPECIFICITY OR TRUE NEGATIVE RATIO. PR: PRECISION OR POSITIVE PREDICTED VALUE). ACC: ACCURACY.

\begin{tabular}{|c|c|c|c|c|c|c|}
\hline \multirow{2}{*}{ Reference } & \multirow{2}{*}{ Input } & \multirow{2}{*}{ Architecture } & \multirow{2}{*}{ Methodology } & \multicolumn{2}{|c|}{ Data set } & \multirow{2}{*}{ Eval. metrics } \\
\hline & & & & Training & Test & \\
\hline $\begin{array}{l}\text { Chen et al. } \\
\text { (2015) [21] }\end{array}$ & $\mathrm{ONH}$ area & Custom (5 CONV, 2 FC) & $\begin{array}{l}\text { Training from scratch with data } \\
\text { augmentation }\end{array}$ & $\begin{array}{l}\text { Sample }(n=99) \text { from } \\
\text { ORIGA / ORIGA }\end{array}$ & ORIGA / Private & AUC \\
\hline $\begin{array}{l}\text { Chen et } \overline{a l} \text {. } \\
\text { (2015) [22] }\end{array}$ & $\mathrm{ONH}$ area & Custom (ALADDIN) & $\begin{array}{l}\text { Training from scratch with data } \\
\text { augmentation }\end{array}$ & $\begin{array}{l}\text { Sample }(n=99) \text { from } \\
\text { ORIGA / ORIGA }\end{array}$ & ORIGA / Private & $\mathrm{AUC}, \mathrm{Se}, \mathrm{Sp}$ \\
\hline $\begin{array}{l}\text { Orlando et al. } \\
\text { (2017) [23] }\end{array}$ & $\begin{array}{l}\text { Cropped } \\
\text { FOV }\end{array}$ & VGG16, Overfeat & $\begin{array}{l}\text { Transferred features and logistic } \\
\text { regression }\end{array}$ & $\begin{array}{l}\text { DRISHTI-GS } \\
\text { (training set) }\end{array}$ & DRISHTI-GS (test set) & AUC \\
\hline $\begin{array}{l}\text { Cerentinia et } \\
\text { al. (2018) [24] }\end{array}$ & $\mathrm{ONH}$ area & GoogLeNet & $\begin{array}{c}\text { Transfer learning with data } \\
\text { augmentation }\end{array}$ & \multicolumn{2}{|c|}{$\begin{array}{l}\text { HRF, RIM-ONE v1, v2 and v3 } \\
\text { (90\% training, } 10 \% \text { test })\end{array}$} & Acc \\
\hline $\begin{array}{l}\text { Christopher et } \\
\text { al. (2018) [25] }\end{array}$ & $\mathrm{ONH}$ area & $\begin{array}{l}\text { VGG16, Inception, } \\
\text { ResNet50 }\end{array}$ & $\begin{array}{l}\text { Training from scratch / Transfer } \\
\text { learning, with data augmentation }\end{array}$ & \multicolumn{2}{|c|}{ Private ( $n=14.822$ ) ( 10 fold cross validation) } & $\mathrm{AUC}, \mathrm{Se}, \mathrm{Sp}$ \\
\hline $\begin{array}{l}\text { Fu } \text { et al. } \\
\text { (2018) [26] }\end{array}$ & $\begin{array}{c}\text { Full } \\
\text { image }\end{array}$ & Custom (DENet) & $\begin{array}{l}\text { Training from scratch with data } \\
\text { augmentation }\end{array}$ & \multicolumn{2}{|c|}{ Private ( $n=1676$ and 5783 ) } & $\begin{array}{l}\text { AUC, Se, Sp, } \\
\text { Balanced Acc }\end{array}$ \\
\hline $\begin{array}{l}\text { Li } \text { et al. } \\
\text { (2018) }\end{array}$ & $\mathrm{ONH}$ area & $\begin{array}{c}\text { AlexNet, VGG16-19, } \\
\text { GoogLeNet, ResNet50-152 }\end{array}$ & Transferred features and SVM & \multicolumn{2}{|c|}{ ORIGA (10 fold cross validation) } & AUC \\
\hline $\begin{array}{l}\text { Li et al. } \\
\text { (2018) }[8]\end{array}$ & $\begin{array}{c}\text { Full } \\
\text { image }\end{array}$ & Inception-v3 & $\begin{array}{c}\text { Training from scratch with data } \\
\text { augmentation }\end{array}$ & Private $(n=31.745)$ & Private $(n=8000)$ & $\begin{array}{l}\text { AUC, Acc, Se, } \\
\text { Sp }\end{array}$ \\
\hline $\begin{array}{l}\text { Liu et al. } \\
\text { (2018) [28] }\end{array}$ & $\mathrm{ONH}$ area & ResNet50 & $\begin{array}{c}\text { Transfer learning with data } \\
\text { augmentation }\end{array}$ & $\begin{array}{l}\text { Sample from private }+ \\
\text { RIM-ONE }(n=3014)\end{array}$ & $\begin{array}{c}\text { Sample from private }+ \\
\text { RIM-ONE }(n=754) / \mathrm{HRF}\end{array}$ & AUC, Se, Sp \\
\hline $\begin{array}{l}\text { Pal et al. } \\
(2018)[29]\end{array}$ & $\mathrm{ONH}$ area & Custom (G-EyeNet) & $\begin{array}{l}\text { Training from scratch with data } \\
\text { augmentation }\end{array}$ & $\begin{array}{c}\text { HRF + RIM-one v3 + } \\
\text { DRISHTI-GS }(n=400)\end{array}$ & DRIONS-DB & AUC \\
\hline $\begin{array}{l}\text { Raghavendra et } \\
\text { al. (2018) [30] }\end{array}$ & $\begin{array}{c}\text { Full } \\
\text { image }\end{array}$ & Custom (4 CONV, 1 FC) & Training from scratch & Private $(n=1003)$ & Private $(n=423)$ & Acc, Se, Sp, Pr \\
\hline $\begin{array}{l}\text { Gómez- } \\
\text { Valverde } \text { et al. } \\
\text { (2019) [31] }\end{array}$ & $\begin{array}{l}\text { Croppped } \\
\text { FOV }\end{array}$ & $\begin{array}{l}\text { VGG19, ResNet, } \\
\text { GoogLeNet }\end{array}$ & $\begin{array}{l}\text { Training from scratch / Transfer } \\
\text { learning, with data augmentation }\end{array}$ & $\begin{array}{c}\text { RIM-ONE }(\mathrm{v} 1, \mathrm{v} 2, \mathrm{v} 3)+ \\
\text { DRISHTI-GS + Private } \\
(n=1560)\end{array}$ & $\begin{array}{c}\text { RIM-ONE (v1, v2, v3) + } \\
\text { DRISHTI-GS + Private } \\
(n=579)\end{array}$ & AUC, $, \mathrm{Se}, \mathrm{Sp}$ \\
\hline
\end{tabular}

TABLE II

OVERVIEW OF EXISTING DEEP LEARNING BASED METHODS FOR OPTIC DISC/CUP SEGMENTATION FROM COLOR FUNDUS PHOTOGRAPHS. DIFFERENT TRAINING/TEST DATA COMBINATIONS ARE SEPARATED WITH BARS ("/"). ONH: OPTIC NERVE HEAD. FOV: FIELD OF VIEW. IOU: INTERSECTION OVER UNION OR JACCARD INDEX. MAE: MEAN ABSOLUTE ERROR OF THE VERTICAL CUP-TO-DISC RATIO. SE: SENSITIVITY, TRUE POSITIVE RATE OR RECALL. SP: SPECIFICITY OR TRUE NEGATIVE RATIO. ACC: ACCURACY.

\begin{tabular}{|c|c|c|c|c|c|c|}
\hline \multirow{2}{*}{ Reference } & \multirow{2}{*}{ Input } & \multirow{2}{*}{ Architecture } & \multirow{2}{*}{ Methodology } & \multicolumn{2}{|r|}{ Data set } & \multirow{2}{*}{ Eval. metrics } \\
\hline & & & & Training & Test & \\
\hline $\begin{array}{l}\text { Lim et al., } \\
2015 \text { [33] }\end{array}$ & $\begin{array}{l}\mathrm{ONH} \\
\text { area }\end{array}$ & Patch-based LeNet & $\begin{array}{l}\text { Vessel segmentation and inpainting, } \\
\text { contrast enhancement on green and red } \\
\text { channels, vessel bending enhancement }\end{array}$ & \multicolumn{2}{|c|}{$\begin{array}{c}\text { MESSIDOR / Private } \\
\text { (cross-validation) }\end{array}$} & IoU, MAE \\
\hline $\begin{array}{l}\text { Zilly et al., } \\
2015 \text { [34] }\end{array}$ & $\begin{array}{l}\mathrm{ONH} \\
\text { area }\end{array}$ & Custom network & $\begin{array}{c}\text { Convolutional filters iteratively learned } \\
\text { using entropy sampling }\end{array}$ & $\begin{array}{l}\text { DRISHTI-GS } \\
\text { (training) }\end{array}$ & DRISHTI-GS (test) & Dice, IoU \\
\hline $\begin{array}{l}\text { Sevastopolsky } \\
2017 \text { 35] }\end{array}$ & $\begin{array}{l}\mathrm{ONH} \\
\text { area }\end{array}$ & Modified U-Net & $\begin{array}{c}\text { Training from scratch on contrast-enhanced } \\
\text { images }\end{array}$ & RIM-ONE v3 & $\begin{array}{l}\text { DRIONS-DB, RIM-ONE } \\
\text { v3, DRISHTI-GS }\end{array}$ & Dice, IoU \\
\hline $\begin{array}{l}\text { Al-Bander et al., } \\
\quad 2018 \text { [36] }\end{array}$ & $\begin{array}{l}\text { Cropped } \\
\text { FOV }\end{array}$ & U-shaped Dense-Net & Training from scratch & ORIGA $(70 \%)$ & $\begin{array}{c}\text { ORIGA }(30 \%), \\
\text { DRIONS-DB, } \\
\text { DRISHTI-GS, ONHSD } \\
\text { and RIM-ONE }\end{array}$ & $\begin{array}{l}\text { Dice, } \text { IoU, } \\
\text { Acc, Se, Sp }\end{array}$ \\
\hline $\begin{array}{l}\text { Edupuganti } \text { et } \\
\text { al., } 2018 \text { [37] }\end{array}$ & $\begin{array}{l}\text { Cropped } \\
\text { FOV }\end{array}$ & U-shaped VGG16 & $\begin{array}{l}\text { Fine tunning (encoder pre-trained on } \\
\text { ImageNet) and data augmentation }\end{array}$ & $\begin{array}{l}\text { DRISHTI-GS } \\
\text { (training) }\end{array}$ & DRISHTI-GS (test) & IoU, Dice \\
\hline 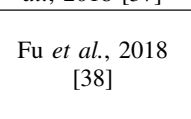 & $\begin{array}{l}\mathrm{ONH} \\
\text { area }\end{array}$ & $\begin{array}{l}\text { Multi-scale input } \\
\text { U-shaped network }\end{array}$ & $\begin{array}{l}\text { Training from scratch on transformed } \\
\text { images in polar coordinates }\end{array}$ & ORIGA $(50 \%)$ & ORIGA $(50 \%)$ & $\begin{array}{c}\text { IoU, } \\
\text { Balanced } \\
\text { Acc, Se, Sp, } \\
\text { MAE }\end{array}$ \\
\hline $\begin{array}{l}\text { Sevastopolsky et } \\
\text { al., } 2018[39]\end{array}$ & $\begin{array}{c}\text { Full } \\
\text { image }\end{array}$ & $\begin{array}{l}\text { U-Net + Residual } \\
\text { U-Net }\end{array}$ & $\begin{array}{l}\text { Two stage networks (pre-segmentation and } \\
\text { refinement) trained from scratch }\end{array}$ & Not specified & $\begin{array}{l}\text { DRIONS-DB, RIM-ONE } \\
\text { v3, DRISHTI-GS, Private }\end{array}$ & Dice, IoU \\
\hline $\begin{array}{l}\text { Sun et al., } \\
2018 \text { [40] }\end{array}$ & $\begin{array}{c}\text { Full } \\
\text { image }\end{array}$ & $\begin{array}{l}\text { ResNet [41] with Faster } \\
\text { R-CNN } \\
\text { connected layers }\end{array}$ & $\begin{array}{l}\text { Training from scratch with data } \\
\text { augmentation for localization, ellipse fitting }\end{array}$ & ORIGA & $\begin{array}{l}\text { ORIGA and SCES } \\
\text { (Private) }\end{array}$ & IoU, MAE \\
\hline
\end{tabular}

however, are based on modifications to the original U-Net architecture [47]. This is due to the fact that this network can achieve good results even when trained using a relatively small amount of images. Architecture changes that heavily increase the capacity of the networks such as those introduced in [37] usually demand the application of transfer learning in the encoding path. In addition, heavy data augmentation through different combination of image transformations has also been explored [38], [40].

\section{Evaluation protocols}

Large discrepancies in the evaluation protocols can be observed in Tables $[$ and II regardless of the target task. These differences are mostly related with two key aspects: (i) the data used for training/evaluation, and (ii) the evaluation metrics.

1) Datasets: Table III summarizes the public available data sets of CFPs for glaucoma classification and/or OD/OC segmentation used by the literature. The REFUGE database (Section III-A) is included for comparison purposes.

In general, we observed that a lack of pre-defined partitions into training and test sets has induced a chaotic practical 
TABLE III

COMPARISON OF THE REFUGE CHALLENGE DATA SET WITH OTHER PUBLICLY AVAILABLE DATABASES OF COLOR FUNDUS IMAGES. QUESTION MARKS INDICATE MISSING INFORMATION, AND N/A STANDS FOR "NOT APPLICABLE".

\begin{tabular}{|c|c|c|c|c|c|c|c|c|c|c|c|}
\hline \multirow{2}{*}{\multicolumn{2}{|c|}{ Dataset }} & \multicolumn{3}{|c|}{ Num. of images } & \multicolumn{3}{|c|}{ Ground truth labels } & \multirow{2}{*}{$\begin{array}{c}\text { Different } \\
\text { cameras }\end{array}$} & \multirow{2}{*}{$\begin{array}{l}\text { Training \& } \\
\text { test split }\end{array}$} & \multirow{2}{*}{$\begin{array}{l}\text { Diagnosis } \\
\text { from }\end{array}$} & \multirow{2}{*}{$\begin{array}{l}\text { Evaluation } \\
\text { framework }\end{array}$} \\
\hline & & Glaucoma & $\begin{array}{c}\text { Non } \\
\text { glaucoma }\end{array}$ & Total & $\begin{array}{c}\text { Glaucoma } \\
\text { classification }\end{array}$ & $\begin{array}{c}\text { Optic } \\
\text { disc/cup }\end{array}$ & $\begin{array}{l}\text { Fovea lo- } \\
\text { calization }\end{array}$ & & & & \\
\hline$\overline{\text { ARIA }}$ 48] & & 0 & 143 & 143 & No & Yes/No & Yes & No & No & $?$ & No \\
\hline DRIONS-DB $\overline{\overline{4}}$ & 49] & - & - & 110 & No & Yes/No & No & $?$ & No & N/A & No \\
\hline DRISHTI-GS [50], & , 51] & 70 & 31 & 101 & Yes & Yes/Yes & No & No & Yes & Image & No \\
\hline DR HAGIS 152 & & 10 & 29 & 39 & Yes & No/No & No & Yes & No & Clinical & No \\
\hline IDRiD 18 & & 0 & 516 & 516 & No & Yes/No & Yes & No & Yes & $?$ & Yes \\
\hline HRF 53] & & 15 & 30 & 45 & Yes & $\mathrm{No} / \mathrm{No}$ & No & No & No & Clinical & No \\
\hline LES-AV 54 & & 11 & 11 & 22 & Yes & $\mathrm{No} / \mathrm{No}$ & No & No & No & Clinical & No \\
\hline ONHSD 55 & & - & - & 99 & No & Yes/No & No & No & No & N/A & No \\
\hline$\overline{\text { ORIGA }} \mid \overline{56}]$ & & 168 & 482 & 650 & Yes & Yes/Yes & No & $?$ & No & $?$ & No \\
\hline RIM-ONE [57] & v1 & 40 & 118 & 158 & Yes & Yes/No & No & No & No & Clinical & No \\
\hline RIM-ONE [57] & v2 & 200 & 255 & 455 & Yes & Yes/No & No & No & No & Clinical & No \\
\hline 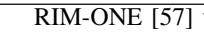 & v3 & 74 & 85 & 169 & Yes & Yes/No & No & No & No & Clinical & No \\
\hline RIGA $[58]$ & & - & - & 750 & No & Yes/Yes & No & Yes & No & $?$ & No \\
\hline REFUGE & & 120 & 1080 & 1200 & Yes & Yes/Yes & Yes & Yes & Yes & Clinical & Yes \\
\hline
\end{tabular}

application of the existing data. As discussed in [19], this affect the feasibility of directly comparing the performance of existing methods, difficulting to conclude which model characteristics are more appropriate to solve each task. To the best of our knowledge, DRISHTI-GS1 [50], [51] is the only existing database for glaucoma assessment that provides a clear training/test split.

Another important aspect is related with the reliability of the assigned diagnostic labels. Some public data sets such as DRISHTI-GS provide glaucoma labels that were assigned based only on image characteristics. This has been also observed in private data sets such as those used in [25] and [8], which were built using images from Internet that were manually graded based on their visual appeareance, without additional clinical information. Surprisingly, no information about the source of the diagnostic labels is provided in most of existing databases (see Table [III). Using images with labels that were not assigned using retrospective analysis of clinical records can be problematic as it might bias automated methods to reproduce wrong labelling practices. On the contrary, clinical labels can aid algorithms to learn and discover other supplemental manifestations of the disease that are still unknown or that are too difficult to distinguish with the naked eye.

The amount of images and their diversity is also an important aspect to consider. In particular, existing databases rarely include images obtained from different acquisitions devices, ethnicities or presenting challenging glaucoma related scenarios. Therefore, the learned models might exhibit a weak generalization ability. To partially bypass this issue, some authors have proposed to train their methods using combinations of different data sets [24], [29].

Finally, it is important to highlight the lack of a large public data set providing both $\mathrm{OD} / \mathrm{OC}$ segmentations and clinical diagnostics simultaneously. ONHSD ${ }^{2}$ [55] and DRIONS-DB ${ }^{3}$ [49] only include segmentations of the OD, and no glaucoma

\footnotetext{
${ }^{1}$ http://cvit.iiitac.in/projects/mip/drishti-gs/mip-dataset2/Home.php ${ }^{2}$ http://www.aldiri.info/Image\%20Datasets/ONHSD.aspx ${ }^{3} \mathrm{http} / / / w w w . i a . u n e d . e s /$ ejcarmona/DRIONS-DB.html
}

labels are given. ARIA $\sqrt[4]{48}$ ] provides OD segmentations and incorporates vessel segmentations and annotations of the fovea center. However, the images correspond to normal subjects and patients with DR and AMD, and no segmentations of the OC are included. DR HAGIS5 [52], HRF 5 [53] and LES-AV7] [54], on the other hand, include reliable diagnostic labels and vessel segmentations, but no labels for the OD/OC. Moreover, their size is relatively small (39, 45 and 22 images, respectively). RIGA ${ }^{8}[58]$ is a recent data set that contains 750 fundus images with OD/OC segmentations but without glaucoma labels. The three releases of RIM-ONE (v1, v2 and v3) [57] provide image-level glaucoma labels and OD segmentations. RIM-ONE v1 and v2 include CFPs cropped around the ONH. Furthermore, RIM-ONE v1 incorporate OD annotations by five different experts and image level labels for control subjects, ocular hypertensive patients and subjects with early, moderate and deep glaucoma. RIM-ONE v2 and $\mathrm{v} 3$, on the contrary, only include OD segmentations by two experts, and the diagnostic labels are classified into normal and glaucoma suspect cases. Moreover, RIM-ONE v3 do not include typical CFPs but stereo images. To the best of our knowledge, only DRISHTI-GS and ORIGA [56] include both glaucoma classification labels and OD/OC segmentations. The diagnostic labels in DRISHTI-GS, however, were assigned solely based on the images [51]. ORIGA, on the other hand, is not publicly available anymore.

2) Metrics: Most of the literature in glaucoma classification uses receiver-operating characteristic (ROC) curves [59] for evaluation, including the area under the curve (AUC) as a summary value [8], [21]-[23], [25], [27]-[29], [31], [38]. Sensitivity and specificity [22], [25], [27], [28], [31], [38] are also used in different studies to complement the AUC when targetting binary classification outcomes. Accuracy was reported in [24], [30] as another evaluation metric, although this

\footnotetext{
${ }^{4}$ https://eyecharity.weebly.com/aria_online.html 5 https://personalpages.manchester.ac.uk/staff/niall.p.mcloughlin/ 6 https://www5.cs.fau.de/research/data/fundus-images/

'https://ignaciorlando.github.io/data/LES-AV.zip

${ }^{8}$ https://deepblue.lib.umich.edu/data/concern/data_sets/3b591905z
} 


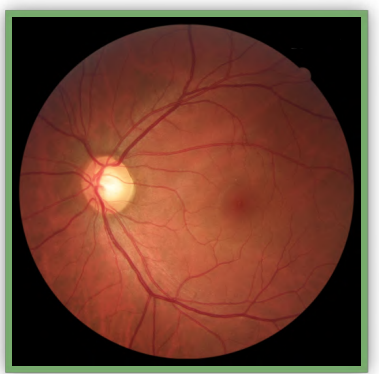

(a) Myopic case

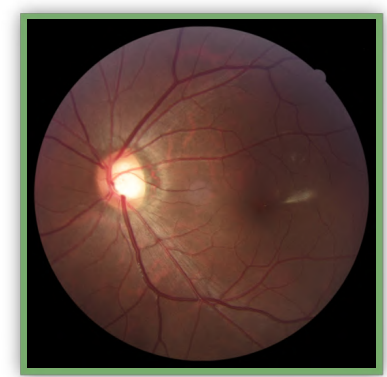

(c) Megalopapilae

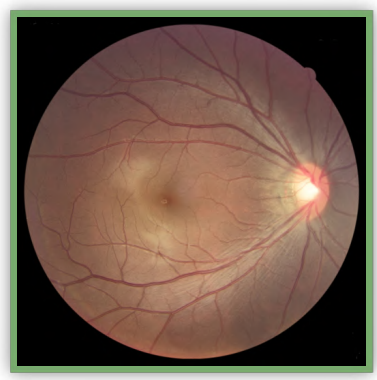

(b) Normal case

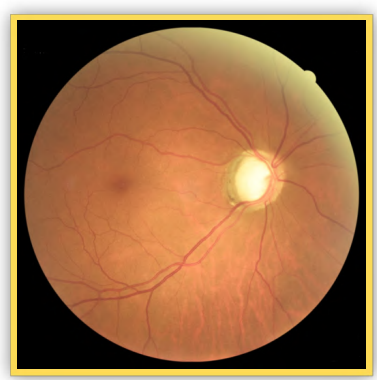

(d) Glaucomatous case
Fig. 3. Representative examples of color fundus photographs from the REFUGE data set. Non-glaucomatous (green) and glaucomatous (yellow) groups. (a) Myopic case with enlarged optic cup. (b) Healthy subject. (c) Patient with megalopapilae. (d, yellow) Glaucomatous case with cupping.

metric might be biased if the proportion of non-glaucomatous images is significantly higher than the glaucomatous ones [60]. To overcome this limitation, Fu et al. [38] used a balanced accuracy, consisting on the average between sensitivity and specificity.

Current literature in OD/OC segmentation make use of classical overlap metrics such as the intersection-over-union (IoU, also known as Jaccard index) [33]-[40] and the Dice index [34]-[37], [39], [40]. Although different by definition, these two metrics can be computed from each other, as they are defined as ratios of overlap between the predicted area and the manual reference annotation [61]. Pixelwise sensitivity and specificity values have been also reported in [36], [38] to illustrate the behavior in terms of false negatives and false positives, respectively. Finally, the accuracy for segmenting both the OD and the OC has been simultaneously assessed by means of the mean absolute error (MAE) of the estimated vs. manually graded CDR values [33], [38], [40].

All these metrics are well-known and were previously used in several domains. However, it is still necessary to come up with a uniform evaluation criteria to assist method comparison and prevent the usage of potentially biased metrics.

\section{The REFUGE CHALLENGE}

\section{A. REFUGE database}

The REFUGE challenge database consists of 1200 retinal CFPs stored in JPEG format, with 8 bits per color channel, acquired by ophthalmologists or technicians from patients sitting upright and using one of two devices: a Zeiss Visucam 500 fundus camera with a resolution of $2124 \times 2056$ pixels
(400 images) and a Cannon CR-2 device with a resolution of $1634 \times 1634$ pixels (800 images). The images are centered at the posterior pole, with both the macula and the optic disc visible, to allow the assessment of the $\mathrm{ONH}$ and potential retinal nerve fiber layer (RNFL) defects. These pictures correspond to Chinese patients $(52 \%$ and $55 \%$ female in validation and test set, respectively) visiting eye clinics, and were retrieved retrospectively from multiple sources, including several hospitals and clinical studies. Only high-quality images were selected to ensure a proper labelling, and any personal and/or device information was removed for anonymization.

Each image in the REFUGE data set includes a reference gold standard glaucomatous/non-glaucomatous label based on its associated clinical record. These diagnostics were restrospectively assigned based on multimodal clinical information, meaning that the decisions were made not only based on the CFP but also with complementary studies such as OCT and visual fields. This ensures the reliability of the classification labels. $10 \%$ of the dataset (120 samples) corresponds to glaucomatous subjects, including Primary Open Angle Glaucoma (POAG) and Normal Tension Glaucoma (NTG). This proportion of diseased cases deviates from the global prevalence of glaucoma $(\approx 4 \%$ for populations aged $40-80$ years [1] $)$. However, reducing the size of the glaucoma set would have negatively affected the ability of the classification approaches to learn features from the diseased cases. Furthermore, in order to model a more representative clinical scenario, the nonglaucomatous set was designed to include not only healthy cases but also patients with other conditions such as myopia or megalopapilae (Fig. 3).

Manual annotations of the OD and the OC were provided by seven independent glaucoma specialists from the Zhongshan Ophthalmic Center (Sun Yat-sen University, China), with 5-10 years of experience in the field. Each of the readers manually drew a tilted ellipse covering the OD and the OC, separately, by using a free annotation tool with capabilities for image review, zoom and ellipse fitting. The final segmentation was created by merging all the annotations using majority voting. The result was a single binary mask per image and region that was further assessed by two experts to ensure its reliability.

Manual pixel-wise annotations of the fovea were also assigned to the images to complement the data set. The fovea position was fixed by 7 independent glaucoma specialists, and a reference standard was created taking the average of these annotations.

The entire set was divided into three fixed subsets: training, validation and test, each of them stratified in such a way that they contain an equal proportion of glaucomatous (10\%) and non-glaucomatous $(90 \%)$ cases. The training set contains all the images acquired with the Zeiss Visucam 500 camera, while the validation and test set includes the lower resolution images captured with the Cannon CR-2 device.

\section{B. Challenge Setup, Evaluation Metrics and Ranking Proce- dure}

REFUGE was held in conjunction with the 5th Ophthalmic Medical Image Analysis (OMIA) workshop, during MICCAI 2018 (Granada, Spain). The challenge proposal was 
accepted after assessing the compliance to good practices proposed in [62], [63]. Thereafter, REFUGE was announced in several platforms to maximize its visibility, including the MICCAI website, its associated mailing lists and on the Grand Challenges in Biomedical Image Analysis website 9. The challenge was officially 1 aunched in $\mathrm{J}$ une $2018 \mathrm{~b}$ y releasing the training set (images and labels) on a dedicated website (https://refuge.grand-challenge.org/Home/). The registered teams were allowed to use the training set to learn and adjust their proposed algorithms for glaucoma classification, OD/OC segmentation and, optionally, for fovea detection. We will not focus on this last task as it was not mandatory for participating on the challenge, and therefore no team submitted results for it on the test set. The registered teams were allowed to use any other public data set for developing their methods, provided that they were easily accessible by everyone.

The validation set (only the images, without labels) was released on July 2018, and the participants were invited to submit their results for an off-line validation. Each participant could receive a maximum of five evaluations on the validation set. Each task was evaluated separately according to a uniform criteria. In particular:

1) Glaucoma classification:: The teams s ubmitted a table with a glaucoma likelihood per each image on the set. A receiver operating characteristics (ROC) curve was created based on the gold standard glaucoma diagnostic, and the area under the curve (AUC) was used as a ranking score for the classification task, $S$ class (the higher, the better). Additionally, a reference sensitivity $\mathrm{Se}=\frac{\mathrm{TP}}{\mathrm{TP}+\mathrm{FN}}$ value at a specificity $\mathrm{Sp}=\frac{\mathrm{TN}}{\mathrm{TN}+\mathrm{FP}}$ of 0.85 was also reported, with TP, FP, TN and FN standing for true/false positives and true/false negatives, respectively. This value was not taken into account for the ranking, but allowed each team to assess the overall performance of the classification algorithm in a setting when a low number of false positives is tolerated.

2) OD/OC segmentation:: The teams submitted one segmentation file for each image. These files were encoded in grayscale BMP format where 0 corresponded to the optic cup, 128 to the optic disc and 255 elsewhere. The results were compared with the gold standard segmentation using the Dice index (DSC) for OD/OC separately, and the mean absolute error (MAE) of the vertical cup-to-disc ratio (vCDR) estimations. In particular, DSC define the overlap between two binary regions:

$$
\operatorname{DSC}_{k}=2 \frac{\left|Y_{k} \cap \hat{Y}_{k}\right|}{\left|Y_{k} \cup \hat{Y}_{k}\right|}
$$

where $Y_{k}$ and $\hat{Y}_{k}$ are the ground truth and predicted segmentations of the region of interest $k$, respectively (with $k=\mathrm{OD}$ or OC). On the other hand, MAE is defined as:

$$
\operatorname{MAE}=\operatorname{abs}\left(\operatorname{vCDR}\left(\hat{Y}_{\mathrm{OC}}, \hat{Y}_{\mathrm{OD}}\right)-\operatorname{vCDR}\left(Y_{\mathrm{OC}}, Y_{\mathrm{OD}}\right)\right)
$$

where $\operatorname{vCDR}(\mathrm{OD}, \mathrm{OC})=\frac{d(\mathrm{OC})}{d(\mathrm{OD})}$ is a function that estimates the vCDR based on the vertical diameter $d$ of the segmentations of the OD and the OC, respectively. Each team was ranked using the average value of each of these metrics separately,

9 grand-challenge.org resulting in three rank values $R_{\mathrm{segm}}^{\mathrm{DSC}}, R_{\mathrm{segm}}^{\mathrm{DSCOC}}$ and $R_{\mathrm{segm}}^{\mathrm{MAE}}$, and an overall segmentation score $S_{\text {segm }}$ was assigned to each team based on the following weighted average:

$$
S_{\mathrm{segm}}=0.35 \times R_{\mathrm{segm}}^{\mathrm{DSCoD}}+0.25 \times R_{\mathrm{segm}}^{\mathrm{DSCOC}}+0.4 \times R_{\mathrm{segm}}^{\mathrm{MAE}}
$$

Notice that in this case, a lower $S_{\text {segm }}$ value is better than a higher one. Since the MAE of the vCDR is calculated based on the segmentation of OC and OD, we set a larger weight for vCDR than to each individual segmentation term. Moreover, it is standard in the literature (Section III) to first segment the OD region and then extract the OC from the cropped OD area. Hence, we assigned a larger weight to the OD segmentation results than to the $\mathrm{OC}$.

An overall validation (off-line) score was assigned to each method based on:

$$
S_{\mathrm{val}}=0.4 \times R_{\text {class }}+0.6 \times R_{\text {segm }}
$$

where $R_{\text {class }}$ and $R_{\text {segm }}$ are the team rank positions based on the classification and segmentation scores $S_{\text {class }}$ and $S_{\text {segm }}$, respectively. A larger weight was assigned to the ranking for the segmentation task as the vCDR, derived from OD/OC segmentation, can be used as a primary score for glaucoma classification. A validation leaderboard was created by setting a rank position $R_{\mathrm{val}}$ for each team, based on $S_{\mathrm{val}}$. Only those teams that submitted reports describing their proposed approaches were taken into account for this leaderboard. These reports can be easily accessed from the challenge website. 10

The first 12 teams according to $S_{\text {val }}$ were invited to attend to the on-site challenge, that was held in person at MICCAI. The test set (only the images) was released during the workshop, and the 12 teams had to submit their results before a time deadline ( 3 hours). The last submission of each team was taken into account for evaluation. Both an on-site rank and a final rank were assigned to each team. The on-site rank $R_{\text {test }}$ was created using the scoring described in Eq. 4. while the final rank $R_{\text {final }}$ was based on a score $S_{\text {final }}$ calculated as the weighted average of the off-line and on-site rank positions:

$$
S_{\text {final }}=0.3 \times R_{\mathrm{val}}+0.7 \times R_{\text {test }}
$$

Notice that a higher weight was assigned to the results on the test set. In this paper we only focus on the results obtained on the test set, during the on-site challenge.

The evaluation was performed using a Python 3.6 opensource framework that was specially developed for the challenge and is publicly available. 11

\section{RESUlts}

This section presents the results on the REFUGE test set of the 12 teams that participated in the on-site challenge. The official final rankings according to the validation and test performances can be accessed on the REFUGE website. We also provide the results obtained by ensembling the top-ranked teams on each task to analyze their complementarity. 
TABLE IV

SUMMARY OF THE GLAUCOMA CLASSIFICATION METHODS EVALUATED IN THE ON-SITE CHALLENGE, IN ALPHABETICAL ORDER USING THE TEAMS NAMES.

\begin{tabular}{|c|c|c|c|c|c|}
\hline Team & Inputs & Architectures & Training set & Methodology & $\begin{array}{c}\text { Post- } \\
\text { processing }\end{array}$ \\
\hline AIML & $\begin{array}{l}\text { Full image / } \\
\text { ONH area }\end{array}$ & $\begin{array}{l}\text { ResNet-50, }-101, \\
-152[41], 38 \text { [64] }\end{array}$ & REFUGE training set & $\begin{array}{c}\text { Ensemble of glaucoma likelihoods from multiple } \\
\text { networks pre-trained on ImageNet and fine-tuned on } \\
\text { REFUGE training set }\end{array}$ & $\begin{array}{l}\text { Ensemble by } \\
\text { averaging }\end{array}$ \\
\hline BUCT & $\begin{array}{l}\text { ONH area, } \\
\text { grayscale }\end{array}$ & Xception 65 & REFUGE training set & Training from scratch on grayscale images & None \\
\hline CUHKMED & $\begin{array}{c}\mathrm{OD} / \mathrm{OC} \\
\text { segmentation }\end{array}$ & None & None & $\begin{array}{l}\text { vCDR values computed from ellipses fitted to } \\
\text { automated OD/OC segmentations }\end{array}$ & None \\
\hline Cvblab & Full image & $\begin{array}{c}\text { VGG19 } \sqrt{66]}, \text { Inception } \\
\sqrt{3}[67], \\
\text { ResNet-50[41], } \\
\text { Xception [65] }\end{array}$ & $\begin{array}{l}\text { REFUGE training set, } \\
\text { DRISHTI-GS, HRF, } \\
\text { ORIGA and RIM-ONE r3 }\end{array}$ & $\begin{array}{l}\text { Ensemble of glaucoma likelihoods from multiple } \\
\text { networks pre-trained on ImageNet and fine-tuned, } \\
\text { classes in REFUGE training set balanced using } \\
\text { SMOTE [68] }\end{array}$ & $\begin{array}{c}\text { Ensemble by } \\
\text { averaging }\end{array}$ \\
\hline Mammoth & $\begin{array}{l}\text { ONH area with } \\
\text { CLAHE }\end{array}$ & $\begin{array}{l}\text { ResNet-18 [41] and } \\
\text { CatGAN [69] }\end{array}$ & $\begin{array}{l}\text { Sample from REFUGE } \\
\text { training set }\end{array}$ & $\begin{array}{l}\text { Ensemble of ResNet models pre-trained on ImageNet } \\
\text { and fine-tuned using REFUGE data and synthetic } \\
\text { images generated with CatGAN }\end{array}$ & None \\
\hline Masker & Full image & ResNet 41] & $\begin{array}{l}\text { REFUGE training set and } \\
\text { ORIGA }\end{array}$ & $\begin{array}{l}\text { Linear combination of vCDR and predictions of } \\
\text { multiple ResNet networks }\end{array}$ & $\begin{array}{c}\text { Ensemble } \\
\text { with vCDR }\end{array}$ \\
\hline NightOwl & $\begin{array}{l}\text { ONH area } \\
\text { with/without } \\
\text { exp. transform }\end{array}$ & Custom & $\begin{array}{l}\text { REFUGE training set } \\
\text { (10-fold cross-validation) }\end{array}$ & $\begin{array}{l}\text { Ensemble of classification networks trained to predict } \\
\text { glaucoma from features produced by the encoders of } \\
\text { the segmentation networks }\end{array}$ & $\begin{array}{l}\text { Ensemble by } \\
\text { maximum }\end{array}$ \\
\hline NKSG & Full image & SENet 70] & $\begin{array}{l}\text { REFUGE training set } \\
\text { (5-fold cross-validation) }\end{array}$ & $\begin{array}{l}\text { SE-Net pretrained on images from Kaggle DR } \\
\text { challenge [17] and fine-tuned on REFUGE data, best } \\
\text { model from cross-validation taken for final prediction }\end{array}$ & None \\
\hline SDSAIRC & $\begin{array}{l}\text { Crop with ONH } \\
\text { in upper-left } \\
\text { corner }\end{array}$ & ResNet-50 41 & REFUGE training set & $\begin{array}{l}\text { Logistic regression classifier trained with vCDR values } \\
\text { from OD/OC segmentation and output of ResNet-50 } \\
\text { model fine-tuned from ImageNet }\end{array}$ & None \\
\hline SmileDeepDR & ONH area & DeepLabv3+ 71] & REFUGE training set & $\begin{array}{l}\text { Adaptation of a segmentation network to predict a } \\
\text { glaucoma likelihood }\end{array}$ & None \\
\hline VRT & $\begin{array}{l}\text { Full image with } \\
\text { custom mask for } \\
\text { attention }\end{array}$ & Custom 72] & $\begin{array}{l}\text { Kaggle [17], } \\
\text { MESSIDOR [73] and } \\
\text { IDRiD [18] }\end{array}$ & $\begin{array}{l}\text { Attention guided model trained on public data sets of } \\
\text { DR images, weakly labelled using pre-trained models } \\
\text { for glaucoma classification, RNFL defects detection and } \\
\text { segmentation of ONH pathological changes }\end{array}$ & None \\
\hline WinterFell & $\mathrm{ONH}$ area & $\begin{array}{l}\text { ResNet-101, }-152 \text { [41], } \\
\text { DensNet-169, -201 } 74]\end{array}$ & ORIGA & $\begin{array}{c}\text { Ensemble of glaucoma likelihoods from multiple } \\
\text { networks pre-trained on Image-Net and fine-tuned on } \\
\text { ORIGA }\end{array}$ & $\begin{array}{l}\text { Ensemble by } \\
\text { mode, max. } \\
\text { and min. }\end{array}$ \\
\hline
\end{tabular}

TABLE V

CLASSIFICATION RESULTS OF THE PARTICIPATING TEAMS IN THE REFUGE TEST SET. THE LAST ROWS CORRESPOND TO THE RESULTS OBTAINED BY THE AVERAGE ENSEMBLE OF THE THREE TOP-RANKED TEAMS AND THE GROUND TRUTH VERTICAL CUP-TO-DISC RATIO (VCDR).

\begin{tabular}{c|c|c|c}
\hline Rank & Team & AUC & $\begin{array}{c}\text { Reference } \\
\text { sensitivity }\end{array}$ \\
\hline $\mathbf{1}$ & VRT & $\mathbf{0 . 9 8 8 5}$ & 0.9752 \\
2 & SDSAIRC & 0.9817 & $\mathbf{0 . 9 7 6 0}$ \\
3 & CUHKMED & 0.9644 & 0.9500 \\
4 & NKSG & 0.9587 & 0.8917 \\
5 & Mammoth & 0.9555 & 0.8918 \\
6 & Masker & 0.9524 & 0.8500 \\
7 & SMILEDeepDR & 0.9508 & 0.8750 \\
8 & BUCT & 0.9348 & 0.8500 \\
9 & WinterFell & 0.9327 & 0.9250 \\
10 & NightOwl & 0.9101 & 0.9000 \\
11 & Cvblab & 0.8806 & 0.7318 \\
12 & AIML & 0.8458 & 0.7250 \\
\hline \multicolumn{2}{|c|}{ Ensemble of top-3 teams } & $\mathbf{0 . 9 9 0 1}$ & $\mathbf{0 . 9 7 6 9}$ \\
\multicolumn{2}{|c|}{ Ground truth vCDR } & 0.9471 & 0.8750 \\
\hline
\end{tabular}

\section{A. Glaucoma classification}

The participating methods for glaucoma classification are summarized in Table IV] Further details about each method are provided in the appendix. The evaluation of the classification task, in terms of AUC and the reference sensitivity at $85 \%$ specificity, is presented in Table $\mathrm{V}$. Two additional approaches are also included: one corresponds to the ensemble of the

\footnotetext{
${ }_{10}$ https://refuge.grand-challenge.org/Results-Onsite_TestSet/

${ }^{11}$ https://github.com/ignaciorlando/refuge-evaluation
}

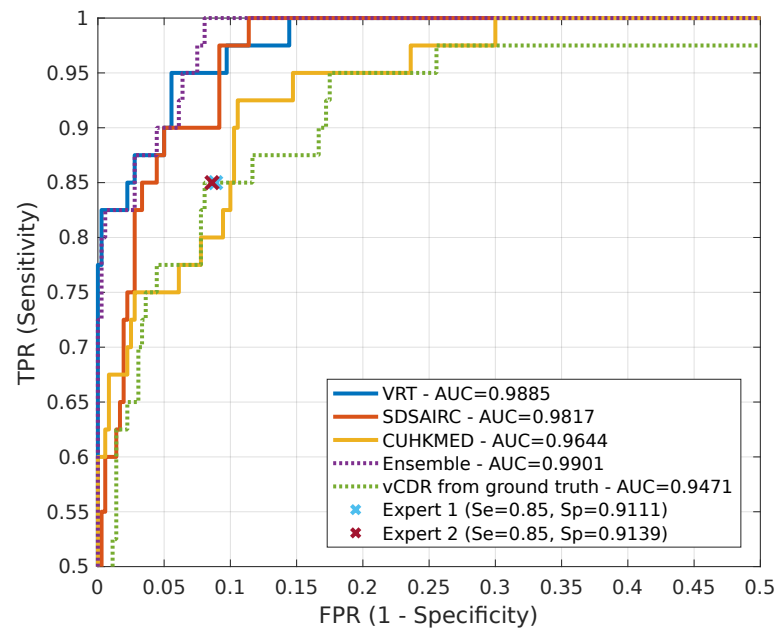

Fig. 4. ROC curves and AUC values corresponding to the three top-ranked glaucoma classification methods (solid lines), their average ensemble (purple dotted line) and the vertical cup-to-disc ratio (vCDR) (green dotted line). Crosses indicate the operating points of two glaucoma experts.

three top-ranked methods, and another one is based on using the ground truth vCDR values as a glaucoma likelihood for classification. The ensemble was built by averaging the scores provided by the teams VRT, SDSAIRC and CUHKMED. To avoid biasing the ensemble towards a single model, each team likelihood was normalized using the maximum value of each 
team. Fig. 4 presents the ROC curves of the three top-ranked teams, their ensemble and the ground truth vCDR values. The curves for each participating method are available for downloading in the challenge website. A series of Matt-Whitney $\mathrm{U}$ hypothesis tests [75] with $\alpha=0.05$ were performed using [76] to study the statistical significance of the differences in the AUC values of the different teams. VRT reported the best classification p erformance, a chieving s ignificantly better results that the ground truth $\mathrm{vCDR}(p=0.006)$. Compared with SDSAIRC and CUHKMED-the second and third teams, respectively-the differences were only significant $\mathrm{w}$ ith respect to CUHKMED (CUHKMED: $p=0.007$, SDSAIRC: $p=0.187$ ). Both SDAIRC and CUHKMED achieved also higher AUC values than the ground truth $\mathrm{vCDR}$, although the differences were not statistically significant $(p>0.05)$. By ensembling the outcomes of the three models, the AUC was marginally improved, with no significant differences with respect to VRT results $(p=0.576)$.

In order to understand the relevance of the classification results, a comparison with glaucoma experts was performed. To this end, two independent glaucoma expert ophtalmologists visually graded the test set images and assigned a binary glaucomatous/non-glaucomatous label to each of them. Notice that no clinical information but only the fundus image was used in this case to perform the annotation. This criteria was followed in order to ensure the same inputs to both the experts and the networks. The sensitivity and specificity values obtained by each human reader are included as expert operating points in Fig. 4. The two points are close to each other due to a high level of agreement between the two experts (96.25\% of the cases). The experts graded with the same sensitivity (85\%) and slightly different specificity $(91.11 \%$ and $91.39 \%$ ) and accuracy $(90.50 \%$ and $90.75 \%)$. If only the cases with their consensus are considered, then their joint accuracy increases to $92.21 \%$, while their joint sensitivity remains the same $(85 \%)$ and the specificity reaches $93.04 \%$.

Fig. 5 illustrates a sample of true negatives, false positives, false negatives and true positive glaucoma detections from the REFUGE test set, as classified by the e nsemble of the three top-ranked models and the two experts. Since the submitted results were not binary decisions but glaucoma likelihoods, the false positive (negative) images were selected such that their assigned value was higher (lower) when the ground truth label was negative (positive). Similarly, the true positive (negative) images correspond to cases in which the ensemble assigned a high (low) glaucoma likelihood. Diagnostic labels reported by the two experts are also included in the figure for comparison purposes.

\section{B. Optic disc/cup segmentation}

The evaluated methods for OD/OC segmentation are briefly described in Table VI. The interested reader could refer to the appendix for further details. The distribution of DSC and MAE values obtained by each of the participating teams in the REFUGE test set are represented as boxplots in Fig. 6. Table VII summarizes the final ranking, based on the average performance of each team. The statistical significance of the differences in performance of the top-ranked teams was assessed by means of Wilcoxon signed-rank tests $(\alpha=$ 0.05). CUHKMED reported the highest DSC values for OD segmentation, significantly outperforming all the alternative approaches $\left(p<1.41 \times 10^{-7}\right)$. VRT and BUCT achieved the second and third higher average DSC values, respectively. However, their performance was not statistical significantly different with respect to each other $(p=0.734)$. For OC segmentation, Masker reported the highest average DSC value, followed by CUHKMED and BUCT. The differences in the DSC values achieved by Masker were statistically significant with respect to every other team $\left(p<1 \times 10^{-4}\right)$, except to CUHKMED ( $p=0.387)$. When evaluating in terms of MAE of the vCDR estimation, Masker also reported the best results, consistently outperforming every other method $(p<0.014)$. CUHKMED retained the second place, although with no significant differences with respect to the BUCT $(p<0.403)$, which was ranked in the third place.

The outputs of the three top-ranked methods in the final leaderboard (CUHKMED, Masker and BUCT) were used to construct an ensemble by majority voting. The results of this combined approach are included in Fig. 6 and Table VII Ensembling these methods allowed to significantly improve their DSC values for OC (Wilcoxon signed rank test, $p<$ $\left.1.91 \times 10^{-7}\right)$ and OD segmentation $\left(p<1.07 \times 10^{-7}\right)$. The estimates of the vCDR obtained from the ensemble segmentations are statistically significantly better than those obtained by CUHKMED and BUCT methods $\left(p<1.27 \times 10^{-4}\right)$ but not with respect to Masker $(p=0.148)$.

Fig. 7 presents the distribution of the DSC values and MAE obtained by the ensemble, stratified according to the glaucomatous/non-glaucomatous ground truth labels of the images. The statistical significance of the differences was evaluated using a Wilcoxon rank-sum test due to the unpaired nature of the two sets (360 vs. 40 samples, respectively). For OD segmentation, the differences in performance between the two groups were not statistically significant $(p=0.3435)$. On the other hand, the ensemble reported higher values for OC segmentation in the glaucomatous group $\left(p=2.09 \times 10^{-5}\right)$. Similarly, the MAE values were significantly smaller in the positive set $(p=0.023)$. An analogous behavior was observed when stratifying the results of each of the three top-ranked methods.

Finally, Fig. 8 presents some qualitative examples of the segmentations of the top-three ranked methods and their ensemble: (a) and (d) present some degree of peripapillary atrophy (PPA), (b) and (c) correspond to cases with ambiguous edges and (c) and (e) are the worst performing cases as measured in terms of DSC for the OD and the OC, respectively. The general behavior of each of the methods is rather stable compared with each other for most of the cases (Fig. 8 (a), (d) and (e)). In challenging scenarios such as those observed in Fig. 8 (b-e), where the edges of the $\mathrm{ONH}$ structures are difficult to assess, ensembling their responses resulted in more accurate segmentations only when the methods were complementary (Fig. 8 (b) and (c) vs. (d) and (e)). 


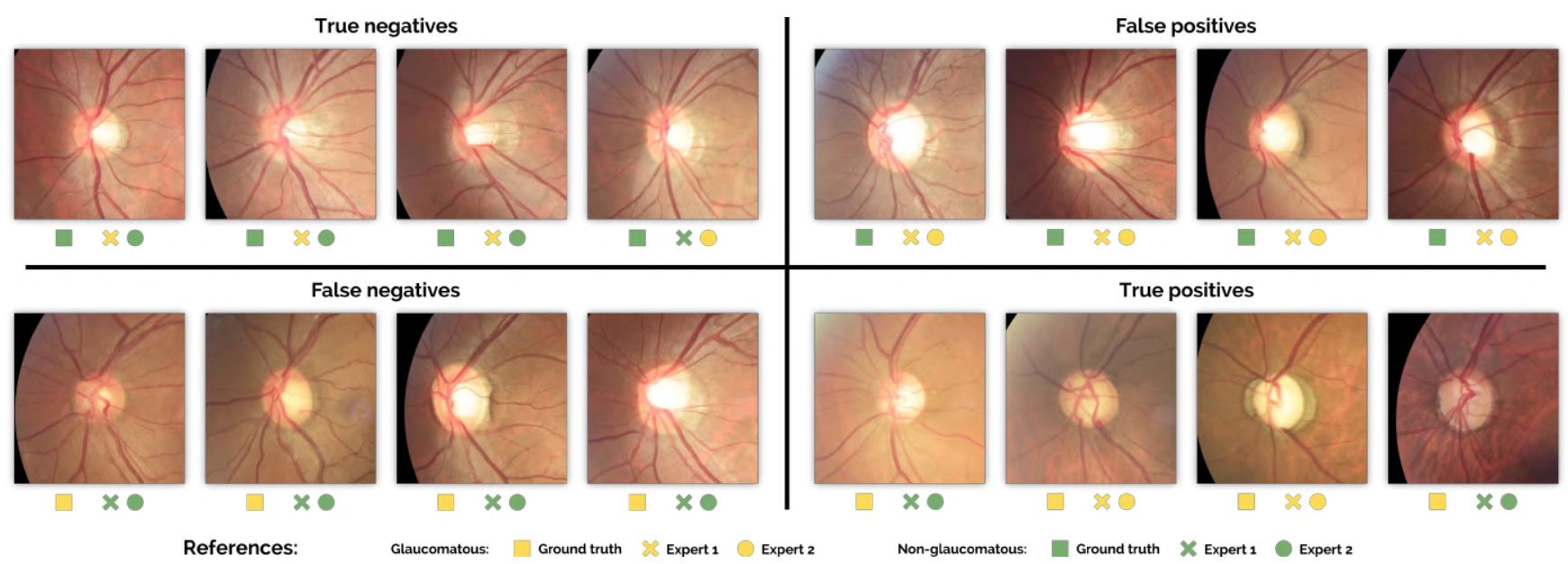

Fig. 5. Qualitative results for glaucoma classification. Images are zoomed in the $\mathrm{ONH}$ area for better visualization. True positives (negatives) correspond to cases in which the ensemble of the three top-ranked methods reported a high (low) score. False positives (negatives) are images for which the ensemble returned a low (high) score. Ground truth and two experts' labels for glaucomatous (yellow) and non-glaucomatous (green) cases are included as colored squares, crosses and circles, respectively.

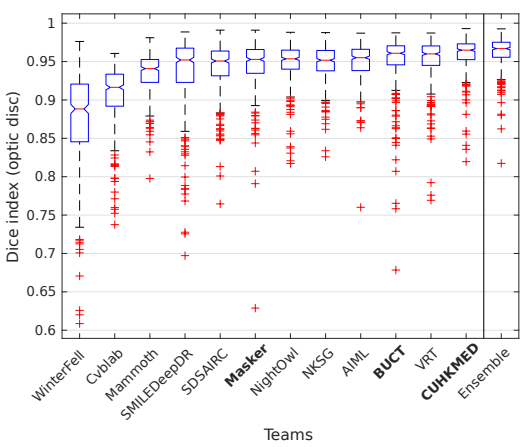

(a) Optic disc segmentation

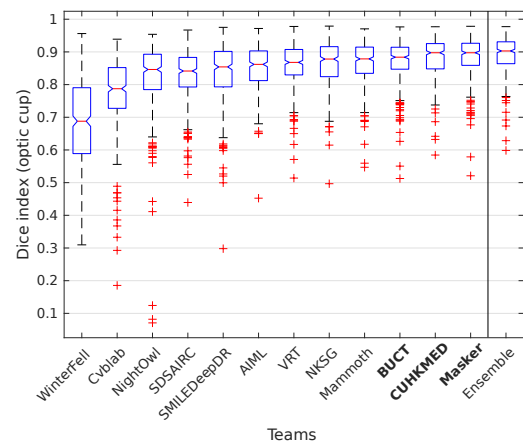

(b) Optic cup segmentation

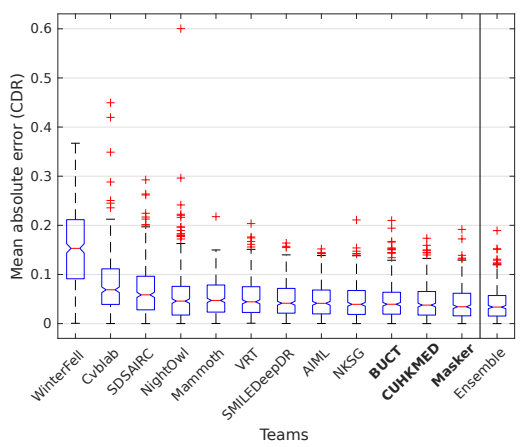

(c) MAE for vCDR

Fig. 6. Box-plots illustrating the performance of each optic disc/cup segmentation method in the REFUGE test set. Distribution of Dice (DSC) values for (a) optic disc and (2) optic cup, and (c) mean absolute error (MAE) of the estimated vertical cup-to-disc-ratio (vCDR). The three top-ranked teams in the final leaderboard (CUHKMED, Masker and BUCT) are highlighted in bolds. Last box on each plot corresponds to the ensemble by majority voting of these methods.
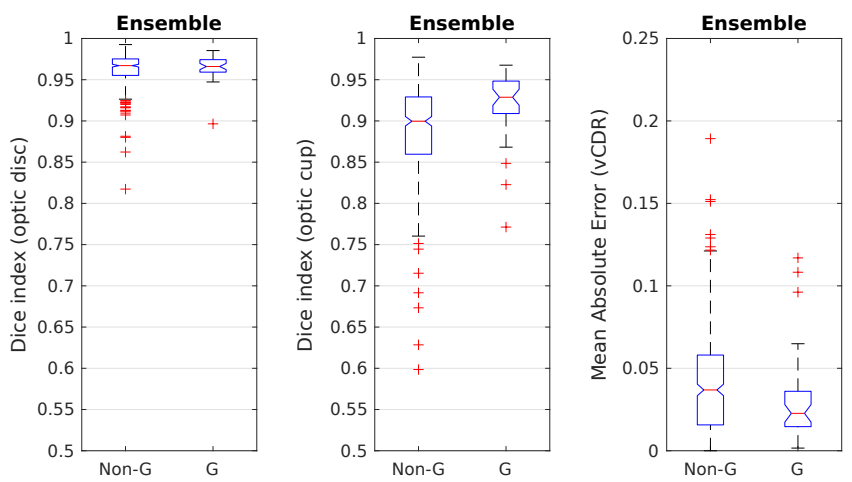

Fig. 7. Results of the ensemble of CUHKMED, Masker and BUCT segmentation methods, stratified for the glaucomatous $(\mathrm{G})$ and non-glaucomatous (Non-G) subsets in the REFUGE test set. From left to right: Dice values for optic disc and optic cup segmentation, and mean absolute error of vertical cup-to-disc ratio (vCDR) estimates.

\section{Discussion}

\section{A. Findings}

1) Classification methods: In line with the evolution of the literature in the field, we observed that the proposed solutions for glaucoma detection were generally based on state-of-theart convolutional neural networks for image classification, with the only exception of SMILEDeepDR and CUHKMED (Table IV). SMILEDeepDR adapted a segmentation network to predict both the OD/OC regions and a glaucoma likelihood, based on the intermediate feature representation generated by the architecture. CUHKMED, on the other hand, proposed to use a normalized vCDR predicted from the OD/OC segmentations.

The classification networks comprised of general-purpose image classification models that were top-ranked in ImageNet Large Scale Visual Recognition Competition [32], such as VGG19 [66], ResNets [41], DenseNets [74], Inception V3 [67] or Xception [65], among others. Since training such deep architectures from scratch on a training set with only 400 
TABLE VI

SUMMARY OF THE GLAUCOMA CLASSIFICATION METHODS EVALUATED IN THE ON-SITE CHALLENGE, IN ALPHABETICAL ORDER USING THE TEAMS NAMES. FCN(S) STANDS FOR FULLY CONVOLUTIONAL NETWORK(S).

\begin{tabular}{|c|c|c|c|c|c|}
\hline Team & Inputs & Architectures & Training set & Methodology & Post-processing \\
\hline AIML & Full image & $\begin{array}{l}\text { FCNs ResNet-50, }-101 \text {, } \\
-152 \text { [41] and }-38 \text { [64] }\end{array}$ & REFUGE training set & $\begin{array}{l}\text { Two stages: (i) Coarse ONH segmentation with } \\
\text { ResNet-50, cropping, (ii) Fine-grain OD/OC } \\
\text { segmentation with multi-view ensemble of networks }\end{array}$ & $\begin{array}{l}\text { Ensemble by } \\
\text { averaging }\end{array}$ \\
\hline BUCT & Full image & U-Net 47] & REFUGE training set & $\begin{array}{l}\text { Two stages: (i) OD segmentation with a U-Net, } \\
\text { postprocessing, cropping (ii) OC segmentation with } \\
\text { U-Net and postprocessing }\end{array}$ & $\begin{array}{l}\text { OD/OC: largest } \\
\text { area element. OD: } \\
\text { ellipse fitting. }\end{array}$ \\
\hline CUHKMED & Full image & $\begin{array}{l}\text { U-Net } 47] \text { and } \\
\text { DeepLabv3+ } 71]\end{array}$ & $\begin{array}{l}\text { REFUGE training set and } \\
\text { validation set (without } \\
\text { labels) }\end{array}$ & $\begin{array}{l}\text { U-Net used for cropping, DeepLabv3+ with geometry } \\
\text { aware loss and domain shift adaptation via adversarial } \\
\text { learning used for final segmentation }\end{array}$ & $\begin{array}{l}\text { Ensemble by } \\
\text { averaging }\end{array}$ \\
\hline Cvblab & $\begin{array}{l}\text { Full image } \\
\text { with } \\
\text { CLAHE }\end{array}$ & Modified U-Net [35] & $\begin{array}{l}\text { DRIONS-DB, } \\
\text { DRISHTI-GS, RIM-ONE } \\
\text { r3 and REFUGE training } \\
\text { set }\end{array}$ & $\begin{array}{l}\text { Two stages: (i) OD segmentation with a modified } \\
\text { U-Net, cropping, (ii) OC segmentation with a modified } \\
\text { U-Net from cropping }\end{array}$ & None \\
\hline Mammoth & Full image & $\begin{array}{c}\text { Mask-RCNN } \overline{77]} \text { and } \\
\text { U-shaped dense } \\
\text { network }\end{array}$ & $\begin{array}{l}\text { Sample from REFUGE } \\
\text { training set }\end{array}$ & $\begin{array}{l}\text { Two stages: (i) OD segmentation with Mask-RNN and } \\
\text { cropping, (ii) OC segmentation with dense U-Net. } \\
\text { Resolution restored with spline interpolation }\end{array}$ & $\begin{array}{l}\text { Ensemble of } \\
\text { outputs, spline } \\
\text { interpolation }\end{array}$ \\
\hline Masker & Full image & Mask-RCNN [7] & $\begin{array}{l}\text { REFUGE training set and } \\
\text { ORIGA }\end{array}$ & $\begin{array}{l}\text { Two stages: (i) Mask-RCNN to identify the ONH area, } \\
\text { cropping, (ii) Ensemble by bootstrap voting of } \\
\text { multiclass Mask-RCNN networks }\end{array}$ & $\begin{array}{l}\text { Ensemble by } \\
\text { voting }\end{array}$ \\
\hline NightOwl & Full image & $\begin{array}{l}\text { U-shaped dense } \\
\text { network }\end{array}$ & REFUGE training set & $\begin{array}{l}\text { Two stages: (i) C-Net for ONH detection, matching } \\
\text { filter and cropping, (ii) OD/OC segmentation using two } \\
\text { F-Nets }\end{array}$ & $\begin{array}{l}\text { Opening and } \\
\text { closing, Gaussian } \\
\text { smoothing }\end{array}$ \\
\hline NKSG & $\mathrm{ONH}$ area & DeepLabv3+ 71] & REFUGE training set & $\begin{array}{l}\text { Multiclass segmentation using DeepLabv3+ on cropped } \\
\text { images pre-processed with pixel quantization }\end{array}$ & None \\
\hline SDSAIRC & Full image & M-Net 38] & REFUGE training set & $\begin{array}{l}\text { Two stages: (i) OD segmentation with M-Net, cropping, } \\
\text { (ii) OC segmentation with M-Net and postprocessing }\end{array}$ & Ellipse fitting \\
\hline SmileDeepDR & Full image & $\begin{array}{l}\text { U-shaped network with } \\
\text { squeeze-and-excitation } \\
\text { blocks (X-Unet) }\end{array}$ & REFUGE training set & $\begin{array}{c}\text { X-Unet pre-trained for predicting ground truth labels, } \\
\text { and fine-tuned separately for segmenting OD/OC using } \\
\text { L1 regression loss }\end{array}$ & None \\
\hline VRT & Full image & $\begin{array}{l}\text { U-Net [47] and } \\
\text { vessel-based } \\
\text { network } 78]\end{array}$ & $\begin{array}{l}\text { IDRiD and RIGA data } \\
\text { sets }\end{array}$ & $\begin{array}{l}\text { Two different U-Nets were applied for OD/OC } \\
\text { segmentation, respectively. An auxiliary CNN using } \\
\text { vessel segmentations as inputs was connected to the } \\
\text { U-Nets to aid in the segmentation }\end{array}$ & $\begin{array}{l}\text { Holes filling, } \\
\text { convex-hull }\end{array}$ \\
\hline WinterFell & Full image & $\begin{array}{l}\text { Faster R-CNN }[79] \text { and } \\
\text { ResU-Net } 80]\end{array}$ & ORIGA & $\begin{array}{c}\text { Two stages: (i) ONH detection with Faster R-CNN, (ii) } \\
\text { OD/OC segmentation in multiple color spaces with } \\
\text { ResU-Net }\end{array}$ & None \\
\hline
\end{tabular}

TABLE VII

OPTIC DISC/CUP SEGMENTATION RESULTS IN THE REFUGE TEST SET. AVERAGE Dice (AVG. DSC) INDEX FOR OPTIC CUP AND DISC AND MEAN ABSOLUTE ERROR (MAE) OF THE VERTICAL CUP-TO-DISC RATIO (VCDR). TEAMS ARE SORTED BY THEIR FINAL RANK. LAST ROW INCLUDES THE RESULTS OBTAINED WITH THE ENSEMBLE BY MAJORITY VOTING OF THE OUTPUTS OF THE THREE TOP-RANKED METHODS IN THE SEGMENTATION LEADERBOARD (CUHKMED, MASKER AND BUCT).

\begin{tabular}{l|c|c|c|c|c|c|c|c}
\hline \multirow{2}{*}{ Rank } & \multirow{2}{*}{ Team } & \multirow{2}{*}{ Score } & \multicolumn{2}{|c|}{ Optic cup } & \multicolumn{2}{c|}{ Optic disc } & \multicolumn{2}{c}{ vCDR } \\
\cline { 4 - 9 } & & & Rank & $\begin{array}{c}\text { Avg. } \\
\text { DSC }\end{array}$ & Rank & $\begin{array}{c}\text { Avg. } \\
\text { DSC }\end{array}$ & Rank & MAE \\
\hline $\mathbf{1}$ & CUHKMED & $\mathbf{1 . 7 5}$ & 2 & 0.8826 & $\mathbf{1}$ & $\mathbf{0 . 9 6 0 2}$ & 2 & 0.0450 \\
2 & Masker & 2.5 & $\mathbf{1}$ & $\mathbf{0 . 8 8 3 7}$ & 7 & 0.9464 & $\mathbf{1}$ & $\mathbf{0 . 0 4 1 4}$ \\
3 & BUCT & 3 & 3 & 0.8728 & 3 & 0.9525 & 3 & 0.0456 \\
4 & NKSG & 4.6 & 5 & 0.8643 & 5 & 0.9488 & 4 & 0.0465 \\
5 & VRT & 5.4 & 6 & 0.8600 & 2 & 0.9532 & 7 & 0.0525 \\
6 & AIML & 5.45 & 7 & 0.8519 & 4 & 0.9505 & 5 & 0.0469 \\
7 & Mammoth & 7.1 & 4 & 0.8667 & 10 & 0.9361 & 8 & 0.0526 \\
8 & SMILEDeepDR & 7.45 & 4 & 0.8367 & 10 & 0.9386 & 8 & 0.0488 \\
9 & NightOwl & 8.6 & 10 & 0.8257 & 6 & 0.9487 & 9 & 0.0563 \\
10 & SDSAIRC & 9.15 & 9 & 0.8315 & 8 & 0.9436 & 10 & 0.0674 \\
11 & Cvblab & 11 & 11 & 0.7728 & 11 & 0.9077 & 11 & 0.0798 \\
12 & WinterFell & 12 & 12 & 0.6861 & 12 & 0.8772 & 12 & 0.1536 \\
\hline \multicolumn{2}{l}{ Ensemble of top-3 teams } & - & $\mathbf{0 . 8 9 2 2}$ & - & $\mathbf{0 . 9 6 2 6}$ & - & $\mathbf{0 . 0 3 9 8}$ \\
\hline
\end{tabular}

images might be prone to overfitting, most of the teams initialized the CNNs with pre-trained weights from ImageNet and fine-tuned them afterwards using the CFPs. Alternatively, NKSG team used pre-trained weights from the Kaggle DR data set [17]. This eases the fine-tuning task as the transition from natural images to fundus photographs is less smooth than the one from images of DR to glaucoma. Only BUCT trained its networks from scratch, although using the $\mathrm{ONH}$ area and not the full images. Nevertheless, we observed that the best solutions were based not only on the application of an existing classification network but also using domain-specific heuristics as discussed next.

CUHKMED achieved the third place by relying only on the prediction of the vCDR. Deep learning was in this case used indirectly, as it was applied for segmenting the OD/OC areas. Exploiting a well-known, clinical parameter such as the vCDR allowed them to identify most of the cases with cupping, which usually correspond to advanced glaucomatous damage. SDSAIRC (second place), on the other hand, obtained better results by combining vCDR estimates with glaucoma likelihoods provided by different CNNs. Team Masker (sixth place) followed a similar idea but using a network trained on full images. Instead, SDSAIRC trained the CNNs using a cropped version of each image in which the $\mathrm{ONH}$ is observed at the upper-left corner. We hypothesize that this configuration indirectly forces the network to identify other complementary signs that are not captured by the vCDR, such as the presence of peripapillary hemorrhages-which appear in the border of the OD (Fig. 2 (b))-or RNFL defects-observed as striated patterns spanning from the ONH (Fig. 2 (c)). The winning team, VRT, further improved this idea by using an attention-guided network that integrated the responses of secondary segmentation models aiming for disease-specific abnormalities. This is a promising way to perform the glaucoma classification task as it resembles to humans targeting glaucoma biomarkers. It is worth mentioning, though, that 

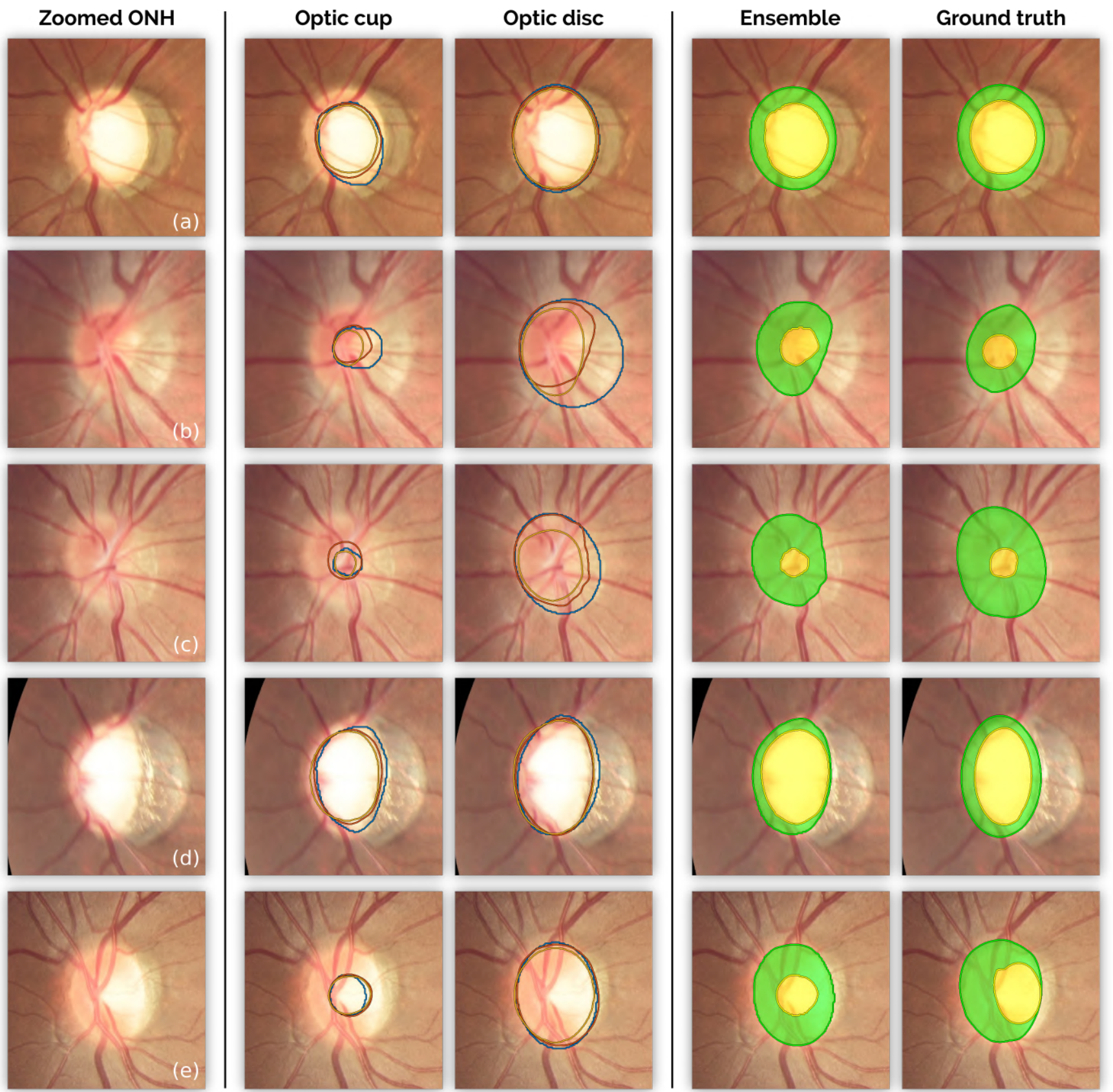

References:
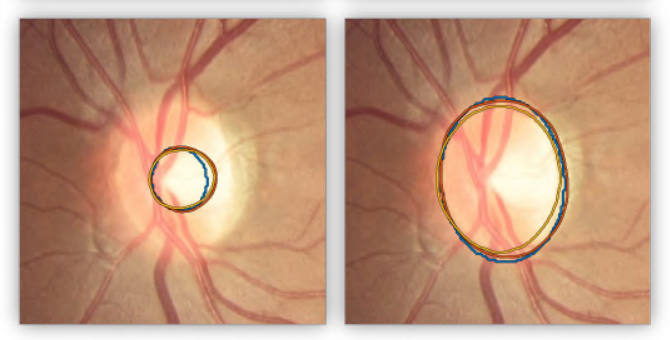

- CUHKMED $=$ Masker - BUCT
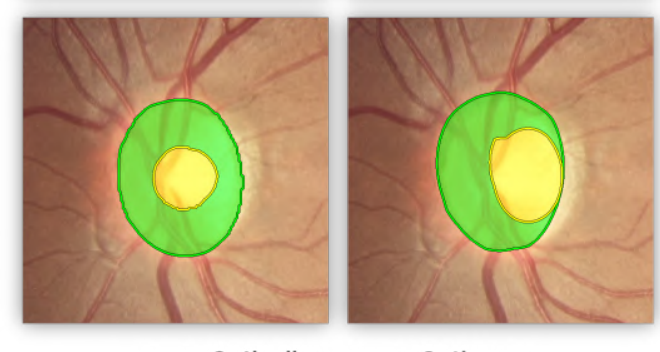

$=$ optic disc $\rightleftharpoons$ optic cup

Fig. 8. Optic disc/cup segmentation results in the REFUGE test set. From left to right: zoomed ONH area, segmentation results from the three top-ranked teams (BUCT, Masker and CUHKMED) for optic cup and disc segmentation, results obtained by ensembling the methods, ground truth segmentations.

such an approach requires manual annotations of lesions in the training set. VRT used pixel-wise weak labels provided by the secondary networks that were independently trained on private data sets. Nevertheless, manual annotations were still needed to train those secondary models. In principle, a classification network with enough capacity would learn to identify abnormal image patterns by itself, although a massive training set size would be needed to this end [81].

2) Segmentation methods: The proposed solutions for OD/OC segmentation were all based on at least one fully convolutional neural network (Table VI). U-shaped networks inspired by the U-Net [47] were the prevalent solutions, although incorporating recent technologies such as residual connections (AIML), atrous convolutions (BUCT) or multiscale feeding inputs (SDSAIRC), among others. Most of the strategies were also based on the two stage approach described in Section II of first roughly identifying the $\mathrm{ONH}$ and then performing the OD/OC segmentation on a cropped version of the original image. The three top-ranked teams followed this principle. CUHKMED and BUCT used a classical U-Net [47] to localize the $\mathrm{ONH}$ area, while Masker applied a Mask-RCNN [77]. Once this area was localized, CUHKMED segmented the OD/OC using a DeepLabv3+ [71] architecture, a recently published approach based on atrous separable convolutions that is 
able to capture multiscale characteristics. Masker, on the other hand, used an ensemble of Mask-RNNs trained with bootstrap, while BUCT used a classical U-Net. NKSG was ranked fourth using the same architecture as CUHKMED, but normalizing image appeareance between training and validation sets using a pixel quantization technique. CUHKMED, on the other hand, accounted for this domain shift using adversarial learning, which could explain its better performance.

Interestingly, we noticed that the three top-ranked methods and their ensemble by majority voting achieved consistently better segmentation results in the subset of glaucomatous subjects than in the non-glaucomatous cases. This can be linked with the fact that advanced glaucoma cases with severe cupping usually present more clear interfaces between the OD and the OC. Such a characteristic would explain why the improvement is more evident in the Dice index obtained for the OC than in the performance for OD segmentation. On the other hand, the segmentation models showed a slightly worst accuracy in challenging scenarios with unclear transitions between the OD/OC, such as those illustrated in Fig. 8 (b), (c) and (e). The lack of depth information in monocular color fundus photographs turns this task significantly difficult. Research in developing automated methods for predicting depth maps from CFPs is currently ungoing, trying to correlate image features with ground truth labels obtained from other imaging modalities such as stereo fundus photography [82] or OCT [83]. These techniques might aid to solve ambiguities in these scenarios.

If the segmentation results are analyzed separately, BUCT and CUHKMED achieved the second and the third place for OC segmentation and the first and third $\mathrm{p}$ laces $\mathrm{f}$ or $\mathrm{OD}$ segmentation, respectively (Table VII). Using the same criteria, Masker achieved the first place for OC s egmentation but the seventh for OD segmentation. Surprinsingly, the team reported the lowest MAE of the vCDR estimation. This indicates that most of their errors in the OD prediction occurs horizontally, and therefore not affect the prediction of its vertical diameter.

3) Ensemble methods: Independently of the target task, we noticed that several submissions exploited to some extent the application of ensembles. Combining the outcomes of multiple models is a common practice in challenges as it allows to achieve (sometimes marginal) quantitative improvements that can eventually ensure higher positions in the final rankings [17], [84]. We observed three types of ensembles in REFUGE. Teams AIML, Cvblab and WinterFell, for instance, combined the outputs of multiple architectures trained with the same data set. This approach allows to take advantage of the characteristics of each model without explicitly integrating them into a single network. Alternatively, team Mammoth averaged the outputs of a single architecture trained under different configurations (e.g. i mages preprocessed w ith multiple strategies). Under this setting, model selection is bypassed as there is no need to choose a single configuration $b$ ecause $a$ subset or even all of them are exploited in test time. Finally, a similar approach was followed by NightOwl and Masker for classification and s egmentation, r espectively, a lthough by training the same architecture on different portions of the training data.
Applying majority voting or averaging on the collective responses of multiple models could certainly ensure more reliable results. This has been recently applied in [85] for retinal OCT analysis with a significant success. We also empirically observed this behavior when ensembling the three top-ranked models for each of the challenge tasks, which resulted in increased performance (Fig. 4 and Fig. 6). This indicates that, despite their impressive but similar performance, the methods are still complementary with each other, and can be integrated to generate a more trustable automated response. This can be qualitatively observed in the segmentation examples in Fig. 8 where e.g. BUCT oversegmented the OD and the OC in (b) but achieved more accurate results in (c). On the other hand, cases such as those in Fig. 8 (d) and (e) illustrate the need of model diversity to achieve more accurate results under challenging conditions.

\section{B. Challenge strengths and limitations}

REFUGE was the first open initiative aiming to introduce a uniform evaluation framework to assess automated methods for OD/OC segmentation and glaucoma classification from CFPs. The challenge provided to the community with the largest public available data set of fundus photographs (1200 scans) to date. In addition, it contains gold standard clinical diagnostic labels, and a high quality reference OD/OC masks and fovea positions from a total of nine glaucoma experts. To the best of our knowledge, the most similar data set to REFUGE was ORIGA [56], which provided 650 images with OD/OC segmentations and glaucoma labels. However, at the time of submitting this manuscript ORIGA was not available anymore ${ }^{12}$, while, more than 350 teams have successfully registered to the REFUGE website to access the database, with 183 requests submitted after the on-site challenge. Such a large interest of the scientific community in accessing REFUGE data clearly demonstrates that a quality open glaucoma data set and challenge was needed.

The challenge design matched most of the principles for evaluating retinal image analysis algorithms proposed in [19]. In particular, REFUGE data set can be easily accessed through a website that is part of the Grand Challenges organization. Furthermore, an automated tool is provided to evaluate the results of any participating team, ensuring a uniform, unbiased criterion for comparing methods, based on trustable and accurate annotations. Furthermore, the data is already partitioned into fixed training, validation and test sets, with labels publicly available only for the first two sets. Future participants are invited to submit their results to the website to estimate their performance on the test set. By keeping these ground truth annotations private we prevent the teams to overfit on test data, ensuring a fair comparison between models.

As the validation set was used to determine which teams were qualified to participate in the on-site challenge, the access to the validation labels was restricted and they were released only after the on-site challenge. Under this constraint, we observed that most of the teams struggled to perform model selection in a uniform setting, and ended up using the

\footnotetext{
12 http://imed.nimte.ac.cn/origa-650.html
} 
REFUGE training set or other third-party data sets. To overcome this issue, we have now publicly released the validation labels. We encourage future participants to use this data not only for model selection but also to perform ablation studies, to empirically demonstrate the contribution of each stage of their pipelines. These experiments might help to identify good practices to follow when designing glaucoma classification and OD/OC segmentation methods.

One limitation of REFUGE is the lack of diverse ethnicities in its data set, as the images correspond to a Chinese population. Ethnicities manifest differently in color fundus photographs due to changes in the pigment of the fundus. Therefore, it cannot be ensured that the best performing models on the REFUGE challenge can be applied on a different population and obtain the same outcomes without retraining. Furthermore, it is worth mentioning that the percentage of glaucoma cases in the REFUGE data set is higher than expected to be encountered in a screening scenario and more representative of a clinical one. A representative screening data set should include comorbidities, diverse ethnicities, ages and genders and low quality images with acquisition artifacts. These characteristics should be addressed in future challenges to ensure that the winning models can be applied in a more general environment.

\section{Clinical implications of the results and future work}

Can we envision automated systems for detecting suspicious cases of glaucoma from fundus photographs? This is still an open question, although REFUGE results might help us to catch a glimpse of a possible answer. With the constant development of much cheaper and easy-to-use fundus cameras, it is expected that this imaging technique will be widespread even more in the decades to follow. Turning it into a costeffective imaging modality for glaucoma screening is still pending due to the subtle manifestation of the early stages of the disease in these images. Nevertheless, novel image analysis techniques based on deep learning can pave the way towards computer-aided screening of glaucoma from fundus photographs.

We observed that some of the proposed segmentation models were able to obtain accurate vertical cup-to-disc ratio estimates. The best team in the segmentation task (CUHKMED) achieved the third place in the classification ranking by using the $\mathrm{vCDR}$ as a glaucoma likelihood, with sensitivity and specificity values almost in pair with two human experts, and statistically equivalent to those obtained using the ground truth measurements. The best performing teams, however, complemented ONH measurements with the classification outcomes of deep learning based models, and were able to significantly surpass the glaucoma experts, with increments in sensitivity up to a $10 \%$. Although these results are limited to a specific image population, we can still argue that these deep learning models are able to identify complementary features, invisible to the naked eye, that are essential to ensure a more accurate diagnosis of the disease. Representing the activation areas on the images might help to better understand which areas were considered by the automated models to produce their predictions. We believe that these tools might contribute in the future to a better identification of glaucoma suspects based on color fundus images alone.

The complementarity of CFP and OCT for automated glaucoma screening still needs to be exploited. Although CFP allows a cost-effective assessment of the retina, features such as the damage in the $\mathrm{ONH}$ or the RNFL are more evident in optic disc centered OCT. This is due to the fact that OCT provides a three dimensional view of the retina, with a micrometric resolution. Hence, the cross-sectional scans-or B-scans-can be used to quantify the thickness of the RNFL or the degree of cupping in the ONH. Nevertheless, the OCT acquisition devices are more expensive than fundus cameras, and the manual analysis of the volumetric information is costly and time-consuming. Developing deep learning methods to quantify glaucoma biomarkers from OCT scans is therefore necessary to complement results in fundus images and pave the way towards cost-effective glaucoma screening.

\section{Conclusions}

We summarized the results and findings from REFUGE, the first open challenge focused on glaucoma classification and optic disc/cup segmentation from color fundus photographs. We analyzed the performance of each of the twelve teams that participated in the on-site edition of the competition, during MICCAI 2018. We observed that the best approaches for glaucoma classification integrated deep learning techniques with well-known glaucoma specific biomarkers such as changes in the vertical cup-to-disc ratio or retinal nerve fiber layer defects. The two top-ranked teams, on the other hand, achieved better results than two glaucoma specialists, a promising sign towards using automated methods to identify glaucoma suspects with fundus imaging. For the segmentation task, the best solutions took into account the domain shift between training and validation sets, aiming to regularize the models to deal with image variability. Cases with ambiguous edges between the optic disc and the optic cup showed to be the most challenging ones. Further research should be performed to improve the results in those scenarios. For both tasks of the challenge, we observed that integrating the outcomes of multiple models allowed to improve their individual performance.

REFUGE unified evaluation framework allowed us to identify good common practices based on the results of the twelve proposed approaches. We expect these findings to help in the future to develop strong baselines for comparison and to aid in the design of new automated tools for image-based glaucoma assessment.

\section{ACKNOWLEDGMENTS}

The financial support by the Christian Doppler Research Association, the Austrian Federal Ministry for Digital and Economic Affairs and the National Foundation for Research, Technology and Development is gratefully acknowledged. J.I.O is funded through the project WWTF AugUniWien/FA7464A0249 (Medical University of Vienna); VRG12009 (University of Vienna). 


\section{REFERENCES}

[1] Y.-C. Tham, X. Li, T. Y. Wong, H. A. Quigley, T. Aung, and C.-Y Cheng, "Global prevalence of glaucoma and projections of glaucoma burden through 2040: a systematic review and meta-analysis," Ophthalmology, vol. 121, no. 11, pp. 2081-2090, 2014.

[2] P. N. Schacknow and J. R. Samples, The glaucoma book: a practical, evidence-based approach to patient care. Springer Science \& Business Media, 2010.

[3] E. Prokofyeva and E. Zrenner, "Epidemiology of major eye diseases leading to blindness in Europe: A literature review," Ophthalmic Research, vol. 47, no. 4, pp. 171-188, 2012.

[4] M. D. Abràmoff, M. K. Garvin, and M. Sonka, "Retinal imaging and image analysis," IEEE Reviews in Biomedical Engineering, vol. 3, pp. 169-208, 2010

[5] U. Schmidt-Erfurth, A. Sadeghipour, B. S. Gerendas, S. M. Waldstein, and H. Bogunović, "Artificial Intelligence in Retina," Progress in Retinal and Eye Research, aug 2018.

[6] P. M. Burlina, N. Joshi, M. Pekala, K. D. Pacheco, D. E. Freund, and N. M. Bressler, "Automated grading of age-related macular degeneration from color fundus images using deep convolutional neural networks," JAMA ophthalmology, vol. 135, no. 11, pp. 1170-1176, 2017.

[7] V. Gulshan, L. Peng, M. Coram, M. C. Stumpe, D. Wu, A. Narayanaswamy, S. Venugopalan, K. Widner, T. Madams, J. Cuadros et al., "Development and validation of a deep learning algorithm for detection of diabetic retinopathy in retinal fundus photographs," JAMA, vol. 316, no. 22, pp. 2402-2410, 2016.

[8] Z. Li, Y. He, S. Keel, W. Meng, R. T. Chang, and M. He, "Efficacy of a deep learning system for detecting glaucomatous optic neuropathy based on color fundus photographs," Ophthalmology, 2018.

[9] M. D. Abràmoff, P. T. Lavin, M. Birch, N. Shah, and J. C. Folk, "Pivotal trial of an autonomous ai-based diagnostic system for detection of diabetic retinopathy in primary care offices," npj Digital Medicine, vol. 1 , no. 1 , p. 39,2018

[10] F. Lavinsky, G. Wollstein, J. Tauber, and J. S. Schuman, "The future of imaging in detecting glaucoma progression," Ophthalmology, vol. 124, no. 12 , pp. S76-S82, 2017.

[11] M. S. Haleem, L. Han, J. van Hemert, and B. Li, "Automatic extraction of retinal features from colour retinal images for glaucoma diagnosis: A review," Computerized Medical Imaging and Graphics, vol. 37, no. 7, pp. $581-596,2013$

[12] A. Almazroa, R. Burman, K. Raahemifar, and V. Lakshminarayanan, "Optic disc and optic cup segmentation methodologies for glaucoma image detection: a survey," Journal of ophthalmology, vol. 2015, 2015.

[13] N. Thakur and M. Juneja, "Survey on segmentation and classification approaches of optic cup and optic disc for diagnosis of glaucoma," Biomedical Signal Processing and Control, vol. 42, pp. 162-189, 2018.

[14] Y. Hagiwara, J. E. W. Koh, J. H. Tan, S. V. Bhandary, A. Laude, E. J. Ciaccio, L. Tong, and U. R. Acharya, "Computer-aided diagnosis of glaucoma using fundus images: A review," Computer Methods and Programs in Biomedicine, 2018.

[15] L. M. Prevedello, S. S. Halabi, G. Shih, C. C. Wu, M. D. Kohli, F. H. Chokshi, B. J. Erickson, J. Kalpathy-Cramer, K. P. Andriole, and A. E. Flanders, "Challenges related to artificial intelligence research in medical imaging and the importance of image analysis competitions," Radiology: Artificial Intelligence, vol. 1, no. 1, p. e180031, 2019.

[16] M. Niemeijer, B. Van Ginneken, M. J. Cree, A. Mizutani, G. Quellec, C. I. Sánchez, B. Zhang, R. Hornero, M. Lamard, C. Muramatsu et al., "Retinopathy online challenge: automatic detection of microaneurysms in digital color fundus photographs," IEEE Transactions on Medical Imaging, vol. 29, no. 1, pp. 185-195, 2010.

[17] Kaggle, "Diabetic Retinopathy Detection," https://www.kaggle.com/ c/diabetic-retinopathy-detection. 2015, [Online; accessed 10-January2019].

[18] P. Porwal, S. Pachade, R. Kamble, M. Kokare, G. Deshmukh, V. Sahasrabuddhe, and F. Meriaudeau, "Indian Diabetic Retinopathy Image Dataset (IDRiD): A Database for Diabetic Retinopathy Screening Research," Data, vol. 3, no. 3, p. 25, 2018.

[19] E. Trucco, A. Ruggeri, T. Karnowski, L. Giancardo, E. Chaum, J. P Hubschman, B. Al-Diri, C. Y. Cheung, D. Wong, M. Abramoff et al., "Validating retinal fundus image analysis algorithms: Issues and a proposal," Investigative Ophthalmology \& Visual Science, vol. 54, no. 5, pp. 3546-3559, 2013

[20] G. Litjens, T. Kooi, B. E. Bejnordi, A. A. A. Setio, F. Ciompi, M. Ghafoorian, J. A. Van Der Laak, B. Van Ginneken, and C. I. Sánchez, "A survey on deep learning in medical image analysis," Medical image analysis, vol. 42, pp. 60-88, 2017.
[21] X. Chen, Y. Xu, D. W. K. Wong, T. Y. Wong, and J. Liu, "Glaucoma detection based on deep convolutional neural network," in 37th Annual International Conference of the IEEE Engineering in Medicine and Biology Society (EMBC). IEEE, 2015, pp. 715-718.

[22] X. Chen, Y. Xu, S. Yan, D. W. K. Wong, T. Y. Wong, and J. Liu, "Automatic feature learning for glaucoma detection based on deep learning," in Medical Image Computing and Computer-Assisted InterventionMICCAI. Springer, 2015, pp. 669-677.

[23] J. I. Orlando, E. Prokofyeva, M. del Fresno, and M. B. Blaschko, "Convolutional neural network transfer for automated glaucoma identification," in Proc. SPIE, vol. 10160, 2017, pp. $101600 \mathrm{U}$ 101 600U-10. [Online]. Available: http://dx.doi.org/10.1117/12.2255740

[24] A. Cerentinia, D. Welfera, M. C. d'Ornellasa, C. J. P. Haygertb, and G. N. Dottob, "Automatic identification of glaucoma using deep learning methods," in MEDINFO 2017: Precision Healthcare Through Informatics: Proceedings of the 16th World Congress on Medical and Health Informatics, vol. 245. IOS Press, 2018, p. 318.

[25] M. Christopher, A. Belghith, C. Bowd, J. A. Proudfoot, M. H. Goldbaum, R. N. Weinreb, C. A. Girkin, J. M. Liebmann, and L. M. Zangwill, "Performance of deep learning architectures and transfer learning for detecting glaucomatous optic neuropathy in fundus photographs," Scientific reports, vol. 8, no. 1, p. 16685, 2018.

[26] H. Fu, J. Cheng, Y. Xu, C. Zhang, D. W. K. Wong, J. Liu, and X. Cao, "Disc-aware ensemble network for glaucoma screening from fundus image," IEEE Transactions on Medical Imaging, vol. 37, no. 11, pp. 2493-2501, 2018.

[27] A. Li, Y. Wang, J. Cheng, and J. Liu, "Combining multiple deep features for glaucoma classification," in 2018 IEEE International Conference on Acoustics, Speech and Signal Processing (ICASSP). IEEE, 2018, pp. 985-989.

[28] S. Liu, S. L. Graham, A. Schulz, M. Kalloniatis, B. Zangerl, W. Cai, Y. Gao, B. Chua, H. Arvind, J. Grigg et al., "A deep learningbased algorithm identifies glaucomatous discs using monoscopic fundus photographs," Ophthalmology Glaucoma, vol. 1, no. 1, pp. 15-22, 2018.

[29] A. Pal, M. R. Moorthy, and A. Shahina, "G-eyenet: A convolutional autoencoding classifier framework for the detection of glaucoma from retinal fundus images," in 2018 25th IEEE International Conference on Image Processing (ICIP). IEEE, 2018, pp. 2775-2779.

[30] U. Raghavendra, H. Fujita, S. V. Bhandary, A. Gudigar, J. H. Tan, and U. R. Acharya, "Deep convolution neural network for accurate diagnosis of glaucoma using digital fundus images," Information Sciences, vol. 441, pp. 41-49, 2018

[31] J. J. Gómez-Valverde, A. Antón, G. Fatti, B. Liefers, A. Herranz, A. Santos, C. I. Sánchez, and M. J. Ledesma-Carbayo, "Automatic glaucoma classification using color fundus images based on convolutional neural networks and transfer learning," Biomedical Optics Express, vol. 10, no. 2, pp. 892-913, 2019

[32] O. Russakovsky, J. Deng, H. Su, J. Krause, S. Satheesh, S. Ma Z. Huang, A. Karpathy, A. Khosla, M. Bernstein et al., "ImageNet large scale visual recognition challenge," International Journal of Computer Vision, vol. 115, no. 3, pp. 211-252, 2015.

[33] G. Lim, Y. Cheng, W. Hsu, and M. L. Lee, "Integrated optic disc and cup segmentation with deep learning," in Tools with Artificial Intelligence (ICTAI), 2015 IEEE 27th International Conference on. IEEE, 2015, pp. $162-169$.

[34] J. G. Zilly, J. M. Buhmann, and D. Mahapatra, "Boosting convolutional filters with entropy sampling for optic cup and disc image segmentation from fundus images," in International Workshop on Machine Learning in Medical Imaging. Springer, 2015, pp. 136-143.

[35] A. Sevastopolsky, "Optic disc and cup segmentation methods for glaucoma detection with modification of u-net convolutional neural network," Pattern Recognition and Image Analysis, vol. 27, no. 3, pp. 618-624, 2017.

[36] B. Al-Bander, B. M. Williams, W. Al-Nuaimy, M. A. Al-Taee, H. Pratt, and Y. Zheng, "Dense fully convolutional segmentation of the optic disc and cup in colour fundus for glaucoma diagnosis," Symmetry, vol. 10, no. 4, p. 87, 2018.

[37] V. G. Edupuganti, A. Chawla, and A. Kale, "Automatic optic disk and cup segmentation of fundus images using deep learning," in 2018 25th IEEE International Conference on Image Processing (ICIP). IEEE, 2018, pp. 2227-2231.

[38] H. Fu, J. Cheng, Y. Xu, D. W. K. Wong, J. Liu, and X. Cao, "Joint optic disc and cup segmentation based on multi-label deep network and polar transformation," IEEE Transactions on Medical Imaging, vol. 37 , no. 7, pp. 1597-1605, 2018.

[39] A. Sevastopolsky, S. Drapak, K. Kiselev, B. M. Snyder, and A. Georgievskaya, "Stack-u-net: Refinement network for image seg- 
mentation on the example of optic disc and cup," arXiv preprint arXiv:1804.11294, 2018.

[40] X. Sun, Y. Xu, M. Tan, H. Fu, W. Zhao, T. You, and J. Liu, "Localizing optic disc and cup for glaucoma screening via deep object detection networks," in Computational Pathology and Ophthalmic Medical Image Analysis. Springer, 2018, pp. 236-244.

[41] K. He, X. Zhang, S. Ren, and J. Sun, "Deep residual learning for image recognition," in Proceedings of the IEEE conference on computer vision and pattern recognition, 2016, pp. 770-778.

[42] S. Ren, K. He, R. Girshick, and J. Sun, "Faster R-cnn: Towards realtime object detection with region proposal networks," in Advances in neural information processing systems, 2015, pp. 91-99.

[43] M. S. Miri, M. D. Abràmoff, K. Lee, M. Niemeijer, J.-K. Wang, Y. H. Kwon, and M. K. Garvin, "Multimodal segmentation of optic disc and cup from sd-oct and color fundus photographs using a machine-learning graph-based approach," IEEE transactions on medical imaging, vol. 34 no. 9, pp. 1854-1866, 2015.

[44] G. D. Joshi, J. Sivaswamy, and S. Krishnadas, "Optic disk and cup segmentation from monocular color retinal images for glaucoma assessment," IEEE Transactions on Medical Imaging, vol. 30, no. 6, pp. 11921205,2011

[45] Y. Xu, L. Duan, S. Lin, X. Chen, D. W. K. Wong, T. Y. Wong, and J. Liu, "Optic cup segmentation for glaucoma detection using lowrank superpixel representation," in International Conference on Medical Image Computing and Computer-Assisted Intervention. Springer, 2014, pp. $788-795$.

[46] Y. LeCun, L. Bottou, Y. Bengio, and P. Haffner, "Gradient-based learning applied to document recognition," Proceedings of the IEEE, vol. 86, no. 11 , pp. 2278-2324, 1998 .

[47] O. Ronneberger, P. Fischer, and T. Brox, "U-net: Convolutional networks for biomedical image segmentation," in International Conference on Medical Image Computing and Computer-Assisted Intervention. Springer, 2015, pp. 234-241.

[48] Y. Zheng, M. H. A. Hijazi, and F. Coenen, "Automated disease/no disease grading of age-related macular degeneration by an image mining approach," Investigative Ophthalmology \& Visual Science, vol. 53 , no. 13, pp. 8310-8318, 2012.

[49] E. J. Carmona, M. Rincón, J. García-Feijoó, and J. M. Martínez-de-la Casa, "Identification of the optic nerve head with genetic algorithms," Artificial Intelligence in Medicine, vol. 43, no. 3, pp. 243-259, 2008.

[50] J. Sivaswamy, S. Krishnadas, G. D. Joshi, M. Jain, and A. U. S. Tabish, "Drishti-gs: Retinal image dataset for optic nerve head (onh) segmentation," in 2014 IEEE 11th International Symposium on Biomedical Imaging (ISBI). IEEE, 2014, pp. 53-56.

[51] J. Sivaswamy, S. Krishnadas, A. Chakravarty, G. Joshi, A. S. Tabish et al., "A comprehensive retinal image dataset for the assessment of glaucoma from the optic nerve head analysis," JSM Biomedical Imaging Data Papers, vol. 2, no. 1, 2015.

[52] S. Holm, G. Russell, V. Nourrit, and N. McLoughlin, "DR HAGIS a fundus image database for the automatic extraction of retinal surface vessels from diabetic patients," Journal of Medical Imaging, vol. 4, no. 1, pp. 014 503-014 503, 2017.

[53] J. Odstrčilík, R. Kolar, A. Budai, J. Hornegger, J. Jan, J. Gazarek, T. Kubena, P. Cernosek, O. Svoboda, and E. Angelopoulou, "Retinal vessel segmentation by improved matched filtering: evaluation on a new high-resolution fundus image database," IET Image Processing, vol. 7 , no. 4, pp. 373-383, 2013

[54] J. I. Orlando, J. Barbosa Breda, K. van Keer, M. B. Blaschko, P. J. Blanco, and C. A. Bulant, "Towards a glaucoma risk index based on simulated hemodynamics from fundus images," in Medical Image Computing and Computer Assisted Intervention - MICCAI 2018, A. F. Frangi, J. A. Schnabel, C. Davatzikos, C. Alberola-López, and G. Fichtinger, Eds. Springer, 2018, vol. 11071, pp. 65-73

[55] J. Lowell, A. Hunter, D. Steel, A. Basu, R. Ryder, E. Fletcher, L. Kennedy et al., "Optic nerve head segmentation," Medical Imaging, IEEE Transactions on, vol. 23, no. 2, pp. 256-264, 2004.

[56] Z. Zhang, F. S. Yin, J. Liu, W. K. Wong, N. M. Tan, B. H. Lee, J. Cheng, and T. Y. Wong, "Origa-light: An online retinal fundus image database for glaucoma analysis and research," in Engineering in Medicine and Biology Society (EMBC), 2010 Annual International Conference of the IEEE. IEEE, 2010, pp. 3065-3068.

[57] F. Fumero, S. Alayón, J. L. Sanchez, J. Sigut, and M. GonzalezHernandez, "Rim-one: An open retinal image database for optic nerve evaluation," in 2011 24th international symposium on computer-based medical systems (CBMS). IEEE, 2011, pp. 1-6.

[58] A. Almazroa, S. Alodhayb, E. Osman, E. Ramadan, M. Hummadi, M. Dlaim, M. Alkatee, K. Raahemifar, and V. Lakshminarayanan,
"Retinal fundus images for glaucoma analysis: the riga dataset," in Medical Imaging 2018: Imaging Informatics for Healthcare, Research, and Applications, vol. 10579. International Society for Optics and Photonics, 2018, p. 105790B.

[59] J. Davis and M. Goadrich, "The relationship between Precision-Recall and ROC curves," in Proceedings of the 23rd international conference on Machine learning. ACM, 2006, pp. 233-240.

[60] J. I. Orlando, E. Prokofyeva, and M. B. Blaschko, "A discriminatively trained fully connected conditional random field model for blood vessel segmentation in fundus images," IEEE Transactions on Biomedical Engineering, vol. 64, no. 1, pp. 16-27, 2017.

[61] A. A. Taha and A. Hanbury, "Metrics for evaluating 3d medical image segmentation: analysis, selection, and tool," BMC medical imaging, vol. 15, no. 1, p. 29, 2015.

[62] A. Reinke, M. Eisenmann, S. Onogur, M. Stankovic, P. Scholz, P. M Full, H. Bogunovic, B. A. Landman, O. Maier, B. Menze et al., "How to exploit weaknesses in biomedical challenge design and organization," in International Conference on Medical Image Computing and ComputerAssisted Intervention. Springer, 2018, pp. 388-395.

[63] L. Maier-Hein, M. Eisenmann, A. Reinke, S. Onogur, M. Stankovic, P. Scholz, T. Arbel, H. Bogunovic, A. P. Bradley, A. Carass et al., "Why rankings of biomedical image analysis competitions should be interpreted with care," Nature Communications, vol. 9, no. 1, p. 5217, dec 2018.

[64] Z. Wu, C. Shen, and A. Van Den Hengel, "Wider or deeper: Revisiting the resnet model for visual recognition," Pattern Recognition, 2019.

[65] F. Chollet, "Xception: Deep learning with depthwise separable convolutions," arXiv preprint, pp. 1610-02 357, 2017.

[66] K. Simonyan and A. Zisserman, "Very deep convolutional networks for large-scale image recognition," arXiv preprint arXiv:1409.1556, 2014.

[67] C. Szegedy, V. Vanhoucke, S. Ioffe, J. Shlens, and Z. Wojna, "Rethinking the inception architecture for computer vision," in Proceedings of the IEEE conference on computer vision and pattern recognition, 2016, pp. $2818-2826$

[68] N. V. Chawla, K. W. Bowyer, L. O. Hall, and W. P. Kegelmeyer, "SMOTE: synthetic minority over-sampling technique," Journal of artificial intelligence research, vol. 16, pp. 321-357, 2002.

[69] S. Wang and L. Zhang, "CatGAN: coupled adversarial transfer for domain generation," arXiv preprint arXiv:1711.08904, 2017.

[70] J. Hu, L. Shen, S. Albanie, G. Sun, and E. Wu, "Squeeze-and-Excitation Networks," in Computer Vision and Pattern Recognition (CVPR), 2018.

[71] L.-C. Chen, Y. Zhu, G. Papandreou, F. Schroff, and H. Adam, "EncoderDecoder with Atrous Separable Convolution for Semantic Image Segmentation," in European Conference on Computer Vision (ECCV), 2018.

[72] J. Son, W. Bae, S. Kim, S. J. Park, and K.-H. Jung, "Classification of Findings with Localized Lesions in Fundoscopic Images Using a Regionally Guided CNN," in Computational Pathology and Ophthalmic Medical Image Analysis. Springer, 2018, pp. 176-184.

[73] E. Decencire, X. Zhang, G. Cazuguel, B. Lay, B. Cochener, C. Trone, P. Gain, R. Ordonez, P. Massin, A. Erginay, B. Charton, and J.-C. Klein, "Feedback on a publicly distributed database: the messidor database," Image Analysis \& Stereology, vol. 33, no. 3, pp. 231-234, Aug. 2014. [Online]. Available: http://www.ias-iss.org/ojs/IAS/article/view/1155

[74] G. Huang, Z. Liu, L. Van Der Maaten, and K. Q. Weinberger, "Densely connected convolutional networks," in Proceedings of the IEEE conference on computer vision and pattern recognition, 2017, pp. 4700-4708.

[75] E. R. DeLong, D. M. DeLong, and D. L. Clarke-Pearson, "Comparing the areas under two or more correlated receiver operating characteristic curves: a nonparametric approach," Biometrics, pp. 837-845, 1988.

[76] I. A. Vergara, T. Norambuena, E. Ferrada, A. W. Slater, and F. Melo, "StAR: a simple tool for the statistical comparison of ROC curves," BMC bioinformatics, vol. 9, no. 1, p. 265, 2008.

[77] K. He, G. Gkioxari, P. Dollár, and R. Girshick, "Mask r-cnn," in Computer Vision (ICCV), 2017 IEEE International Conference on. IEEE, 2017, pp. 2980-2988.

[78] J. Son, S. J. Park, and K.-H. Jung, "Retinal vessel segmentation in fundoscopic images with generative adversarial networks," arXiv preprint arXiv:1706.09318, 2017.

[79] R. Girshick, "Fast r-cnn," in Proceedings of the IEEE international conference on computer vision, 2015, pp. 1440-1448.

[80] S. M. Shankaranarayana, K. Ram, K. Mitra, and M. Sivaprakasam, "Joint optic disc and cup segmentation using fully convolutional and adversarial networks," in Fetal, Infant and Ophthalmic Medical Image Analysis. Springer, 2017, pp. 168-176.

[81] R. Poplin, A. V. Varadarajan, K. Blumer, Y. Liu, M. V. McConnell, G. S. Corrado, L. Peng, and D. R. Webster, "Prediction of cardiovascular 
risk factors from retinal fundus photographs via deep learning," Nature Biomedical Engineering, vol. 2, no. 3, p. 158, 2018.

[82] S. M. Shankaranarayana, K. Ram, K. Mitra, and M. Sivaprakasam, "Fully convolutional networks for monocular retinal depth estimation and optic disc-cup segmentation," arXiv preprint arXiv:1902.01040, 2019.

[83] M. J. Thurtell, R. H. Kardon, and M. K. Garvin, "Local estimation of the degree of optic disc swelling from color fundus photography," Computational Pathology and Ophthalmic Medical Image Analysis, vol. 11039, p. 277, 2018.

[84] K. Kamnitsas, W. Bai, E. Ferrante, S. McDonagh, M. Sinclair, N. Pawlowski, M. Rajchl, M. Lee, B. Kainz, D. Rueckert et al., "Ensembles of multiple models and architectures for robust brain tumour segmentation," arXiv preprint arXiv:1711.01468, 2017.

[85] J. De Fauw, J. R. Ledsam, B. Romera-Paredes, S. Nikolov, N. Tomasev, S. Blackwell, H. Askham, X. Glorot, B. ODonoghue, D. Visentin et al., "Clinically applicable deep learning for diagnosis and referral in retinal disease," Nature medicine, p. 1, 2018.

[86] S. Wang, L. Yu, X. Yang, C.-W. Fu, and P.-A. Heng, "Patch-based output space adversarial learning for joint optic disc and cup segmentation," IEEE transactions on medical imaging, 2019.

[87] M. Sandler, A. Howard, M. Zhu, A. Zhmoginov, and L.-C. Chen, "Mobilenetv2: Inverted residuals and linear bottlenecks," in Proceedings of the IEEE Conference on Computer Vision and Pattern Recognition, 2018, pp. 4510-4520.

[88] T.-Y. Lin, M. Maire, S. Belongie, J. Hays, P. Perona, D. Ramanan, P. Dollár, and C. L. Zitnick, "Microsoft COCO: Common objects in context," in European conference on computer vision. Springer, 2014, pp. 740-755.

[89] D. Berman, S. Avidan et al., "Non-local image dehazing," in Proceedings of the IEEE conference on computer vision and pattern recognition, 2016, pp. 1674-1682.

[90] Z. Farbman, R. Fattal, D. Lischinski, and R. Szeliski, "Edge-preserving decompositions for multi-scale tone and detail manipulation," in ACM Transactions on Graphics (TOG), vol. 27, no. 3. ACM, 2008, p. 67.

[91] L.-C. Chen, G. Papandreou, F. Schroff, and H. Adam, "Rethinking atrous convolution for semantic image segmentation," arXiv preprint arXiv:1706.05587, 2017.

\section{APPENDIX \\ PARTICIPATING METHODS}

\section{A. AIML}

1) OD/OC segmentation: The method was a two-stage approach based on a combination of multiple dilated fullyconvolutional networks (FCNs) based on ResNet-50, -101, -152 [41] and -38 [64]. First, a ResNet-50 FCN was used to coarsely segment the $\mathrm{ONH}$. The corresponding region was afterwards cropped to cover approximately one quarter of the original resolution. These images were used to fed the ResNet$50,-101,-152[41]$ and -38 [64] models, which produced the final segmentations of the OD/OC. The networks were trained using the REFUGE training set with data augmentation, including rescalings and rotations. The final prediction was obtained by averaging multi-view results produced by all the networks on different augmented versions of each image.

2) Glaucoma classification: Two sets of classification models were combined. One was trained using the whole fundus images, while the other was trained using only local regions around the $\mathrm{ONH}$. The OD/OC area was detected using the segmentation model described above. Subsequently, the REFUGE training set was used to fine-tune pre-trained ResNet-50, -101, -152 [41] and -38 [64] models. The final classification result was assigned by ensembling the outputs of these architectures by averaging.

\section{B. $B U C T$}

1) OD/OC segmentation: The OD/OC were segmented separately by two different U-Net [47] models. First, the images on the REFUGE training set were resized to fit the resolution of those on the validation set and converted to gray scale. Then, for OD segmentation, a square of $817 \times 817$ pixels was cropped from the input images, leaving the $\mathrm{ONH}$ on the left-hand side, and then resized to $256 \times 256$ pixels. A U-Net with less convolutional filters than the original approach [47] was applied to retrieve the OD. To remove false positives, the largest connected component was taken, and an ellipse was fitted to the OD segmentation. For OC segmentation, the smallest rectangle containing the OD was clipped out, and each side of the rectangle was extended with 100 pixels to fit a resolution of $128 \times 128$ pixels. The same U-Net architecture was retrained then on these images and applied to retrieve the OC. The largest connected component was taken as the final result, too. In both cases, the U-Nets were trained using the REFUGE training set with data augmentation, including rotations and flippings.

2) Glaucoma classification: The same cropping strategy applied for OD/OC segmentation was used for this task. The resulting CFPs were then transformed into grayscale images. Standard data augmentation techniques such as rotations and shiftings were applied to increase the size of the training set. Then, an X-ception [65] network was trained from scratch for glaucoma classification using grayscale versions of the color images on the REFUGE training set and the ground truth annotations.

\section{CUHKMED}

1) OD/OC segmentation: A patch-based Output Space Adversarial Learning framework (pOSAL) [86] was introduced for this task. This method enables output space domain adaptation to reduce the segmentation performance degradation on target datasets with domain shift. A region of interest (ROI) containing the OD from each original image was first extracted using a U-Net [47] model. The DeepLabv3+ [71] architecture was afterwards applied for segmentation, using the backbone of MobileNetV2 [87]. Considering the shape of the OD and OC, a morphology-aware segmentation loss was designed to force the network to generate smooth predictions. To overcome the domain shift between training and testing datasets, adversarial learning was exploited, encouraging the segmentation predictions in the target domain to be similar to the source ones. Specifically, a patch-based discriminator was used to distinguish whether the prediction came from the source or the target domain. The final image prediction was acquired by ensembling five models, to further improve the segmentation performance.

2) Glaucoma classification: This task was tackled without using a dedicated method. Instead, the authors proposed to use the OD/OC segmentation masks-automatically obtained with the method described below-to compute the vertical CDR (vCDR). To this end, two ellipses were fitted to the the OD and OC masks, respectively. The vCDR values were normalized into $0-1$ as a final classification probability following: $p_{\text {new }}=$ 
$\frac{p-p_{\min }}{p_{\max }-p_{\min }}$, where $p$ is the calculated vCDR values, $p_{\min }$ and $p_{\max }$ are the minimum and maximum vCDR values among all the testing images.

\section{Cvblab}

1) $O D / O C$ segmentation: A two-stage process was followed for this task, based on a modified U-Net architecture [35]. The OD was segmented first and the resulting mask was used to crop the image and segmenting the OC. As a pre-processing technique, the Contrast Limited Adaptive Histogram Equalization (CLAHE) method, was applied. The images were also resized to $256 \times 256$ pixels before feeding the network. The models were trained using DRIONS-DB, DRISHTI-GS, RIM-ONE v3 and the REFUGE training set.

2) Glaucoma classification: An ensemble of VGG19 [66], GoogLeNet (InceptionV3) [67], ResNet-50 [41] and the Xception [65] architectures was applied for this task. Each network was independently fine-tuned from the weights pre-trained from ImageNet [32] to identify glaucomatous images, using DRISHTI-GS1, HRF, ORIGA, RIM-ONE and the training set of the REFUGE databases. Data augmentation was applied in the form of vertical and horizontal flippings, rotations up to $50^{\circ}$, height/width shifts of 0.15 and zooms in a range between 0.7 and 1.3. Prior to fine tunning, the training data was balanced using SMOTE [68] on the REFUGE training set, with the aim of reducing the bias on the prediction model towards the more common class (Normal). All the images were resized to $256 \times 256$ pixels before feeding the network. The results were merged together by ensembling the models' outputs taking the average glaucoma likelihood.

\section{E. Mammoth}

1) OD/OC segmentation: A Mask-RCNN [77] and a Dense U-Net [47] were ensembled for this task. For Mask-RCNN, the OD was first segmented. Then, each input image was cropped around its center to retrieve a patch with a size of $512 \times 512$ pixels, and the segmentation of the OC was performed on it. For the Dense U-Net, which is a modified U-Net architecture with dense convolutional blocks and dilated convolutions, the OD was first segmented. Then the probability mask was used as additional channel of the input (as attention) to segment OC. Both networks were trained using a linear combination of cross-entropy and Dice losses. The probability outputs of both networks were averaged to generate the final segmentation results. A subsample from the original REFUGE training set was used to learn the models. In particular, it was divided into two new sets, one used for training (32 glaucoma images and 288 non-glaucoma images) and a second for validation (8 glaucoma images and 72 non-glaucoma images). The MaskRCNN internally used a ResNet-50 [41] model pre-trained in the COCO [88] data set and fine-tuned using the above mentioned training set.

2) Glaucoma classification: The OD/OC segmentation method was used to crop each input image and generate a patch centered in the $\mathrm{ONH}$, covering 1.5 times the radius of the OD. The resulting image was then resized to $448 \times 448$, and CLAHE contrast equalization and mean color normalization were subsequently applied to uniform image characteristics across data sets. A combination of a ResNet-18 [41] (supervised) and a CatGAN [69] (semi-supervised) classification networks was applied for diagnosis. The CatGAN was used to aid the learning process of the ResNet-18 model in a semi-supervised setting, using fake images generated by the CatGAN to increase the size of the training set. The same training/validation partition used for OD/OC segmentation was applied for this task. A series of ResNet-18 models was trained using 4-fold cross-validation on these training set and a weighted and an unweighted cross-entropy loss, resulting in $4 \times 2=8$ models in total. At inference time, the predictions of all the models were averaged into a final glaucoma likelihood.

\section{F. Masker}

1) OD/OC segmentation: The first step consisted of localizing the ONH region. A Mask-RCNN [77] architecture was used to this end. Afterwards, the image was cropped around the $\mathrm{ONH}$ to build a new training set. This set was divided into 14 partitions based on a bagging principle. Different image preprocessing techniques were applied to each subset, namely image dehazing [89] and edge-preserving multiscale image decomposition based on weighted least squares optimization [90]. Different networks including Mask-RCNN [77], U-Net [47] and M-Net [38] were trained on each subset, and the final result was obtained by a voting procedure in which regions predicted by $80 \%$ of all the networks were taken as the final segmentation.

2) Glaucoma classification: The vCDR value was first calculated using the segmentation results obtained with the previously described method. Subsequently, several classification networks based on ResNet [41] were trained from scratch to predict the risk of glaucoma. The REFUGE training set and ORIGA were used to learn the models. The final result was obtained based on a linear combination of the vCDR values and the prediction of the classification networks. We use ResNet-50, ResNet-101 and ResNet-152 as the basic classification models. The final glaucoma risk is:

$$
\text { Glaucoma Risk }=0.8 \times \mathrm{CDR}+0.2 \times \text { CNets } .
$$

Here, CDR is the vertical cup to disc ratio and CNets is the final voting of the ensemble classification networks. If $80 \%$ of all the networks predict a image with high risk of glaucoma, CNets $=1$, otherwise, CNets $=0$. In our implementation, we use 14 different networks.

\section{G. NightOwl}

1) $O D / O C$ segmentation: A coarse to fine approach was proposed for this task, based on two dense U-shaped networks with dense blocks [74], namely CoarseNet (C-Net) and FineNet (F-Net), respectively. The C-Net model was used to coarsely localize the ONH region. Then, the F-Net was applied to retrieve the final segmentation of the OD and the OC. A modified version of pooling based on the mean of average and max-pooling was applied for better feature accumulation. The images were preprocessed using histogram matching-to normalize the intensities in the sample space-and exponential 
transformations-to enhance the boundaries of the optic cup- Standard data augmentation techniques were applied to the REFUGE training set to balance the number of images from each class (glaucomatous / non-glaucomatous). The original inputs, resized to $112 \times 112$ pixels, were fed to the C-Net for localizing the $\mathrm{ONH}$ region. This area was then extracted from the original input image, resized to $112 \times 112$ pixels too, and fed to two different F-Nets for OD/OC segmentation. Outliers were removed using morphological operations (opening and closing) and Gaussian smoothing.

2) Glaucoma classification: T he e ncoders of e ach F-Net were used for extracting two vectors of 2048 features each, one for the OD and one for the OC. Dimensionality reduction via convolutions was applied to retrieve two new vectors with 64 features each. The concatenation of these two vectors was used to feed a neural network with 4 fully connected layers, trained to predict the glaucoma likelihood. The weights of the F-Net encoders were not adjusted for glaucoma classification, only the weights used for dimensionality reduction and those of the fully connected layers. 10-fold cross-validation was applied to retrieve 10 different models, and 7 of them were retrieved based on their confusion matrices. The final glaucoma likelihood was obtained by taking the maximum likelihood from all the models.

\section{H. NKSG}

1) OD/OC segmentation: The DeepLabv3+ [71] architecture was used for this task, based on the assumption that atrous spatial pyramid pooling (ASPP) is effective to segment objects at multiple scales. The network was trained using crossentropy as the loss function. The images were pre-processed using pixel quantization to reduce the sensitivity of the model to changes in color and to improve its robustness. Moreover, the segmentation approach was applied on cropped versions of the input images. These were obtained by extracting a bounding box surrounding the $\mathrm{ONH}$ area.

2) Glaucoma classification: This task was performed using a SENet [70] architecture. This network has large capacity, as it has 154 layers in total. Instead of using fully connected layers, it uses $1 \times 1$ convolutions. The images were preprocessed by applying the same strategy used for segmentation. The glaucomatous/non-glaucomatous classes were balanced using re-sampling. By means of data augmentation using rotations and stretching, the REFUGE training set was increased to a total of 2000 images.

\section{SDSAIRC}

1) OD/OC segmentation: A method inspired by the MNet [38] was applied for this task. An area of $480 \times 480$ pixels size was defined and prepared as the segmentation ROI for each image, centered on the OD and transformed to polar coordinates afterwards. The histogram of the test images were matched to the average histogram of the REFUGE training set to compensate image variance per camera vendor. The segmentation task was divided into OD segmentation from the segmentation ROI and OC segmentation from the bounding box of the OD. This box was tightly cropped to contain the entire OD. This two stage separation helped to tackle the difficulty in finding the ideal weights for the M-Net [38]. The segmentation accuracy was further improved by postprocessing the resulting masks using ellipse fitting.

2) Glaucoma classification: A ResNet-50 [41] network with pre-trained weights from ImageNet [32] was fine-tuned on the REFUGE training for glaucoma classification. Histogram matching was applied to uniform the appeareance of images with respect to the training set. The CFPs were also cropped in such a way that the OD was positioned in the upper-left corner. This setting allows to capture RNFL defects in more detail than cropping a square centered in the $\mathrm{ONH}$. The final glaucoma likelihood was obtained by averaging the classification score predicted by the network with the resulting score of a logistic regression which takes advantage of vCDR value, estimated from the OD/OC segmentation, as an input. To this end, the logistic regression classifier was trained separately using the transformed vCDR value.

\section{J. SMILEDeepDR}

1) OD/OC segmentation: A modified U-Net [47] architecture, namely X-Unet, was applied for this task. It used 3 inputs so that it was able to receive more original raw pixel information during training. This strategy was used to reduce the risk of overfitting while enhancing the network's learning capability. Moreover, squeeze-and-excitation blocks were embedded into this U-Net variant to weight the features from different convolutional layers' channels. Such a mechanism was able to selectively amplify the valuable channel-wise features and suppress the useless feature from global information. In addition, deconvolution were used in the network decoder to refine the decoding capability by refusing the features between different level encoded features and the corresponding level decoded features. The segmentation task was also posed as a linear regression task instead of a typical pixel classification problem, using $L_{1}$ loss for training. A splitcopy-merge strategy was followed: a X-Unet network was trained first to predict the ground labels. Secondly, two XUnets were separately fine-tuned using the learned weights, only to predict the OD and the OC, respectively. Then, the predictions of both networks were merged to get the final result.

2) Glaucoma classification: The Deeplabv3+ [91] was modified and used as a classifier. Its last layer was replaced by a global average pooling layer followed by a fully connected layer. The model was trained on the REFUGE training set using the cross-entropy loss. Instead of using the full images, a pre-processing stage based on cropping the regions around the ONH was followed.

\section{K. $V R T$}

1) OD/OC segmentation: A U-Net [47] based architecture was used, complemented by an auxiliary $\mathrm{CNN}$ [78] that took a vessel segmentation mask and generated a coarse mask with the estimated OD/OC location. The output of the second network was concatenated to the bottleneck layer of the UNet to generate the final segmentation mask. A combined loss 
$L_{\text {total }}=L_{\text {main }}+\lambda * L_{\text {vessel }}$ was applied, where $L_{\text {main }}$ and $L_{\text {vessel }}$ are pixel-wise binary cross entropy for the U-Net and the auxiliary CNN. The values for $\lambda$, the depth of U-Net and the number of filters a $t \mathrm{t}$ he 1 ast $l$ ayer of $t$ he a uxiliary $\mathrm{CNN}$ were experimentally selected using a hill-climbing approach. The OD and the OC were segmented separately using two different U-Net architectures. Holes in the final segmentations were filled, and the OD/OC a reas w ere c onverted to convexhulls to ensure a single binary mask per regions.

2) Glaucoma classification: $\mathrm{T}$ he $\mathrm{m}$ ethod $\mathrm{w}$ as $\mathrm{b}$ ased on three architectures as described in $[72]^{13}$, each of them targetting glaucoma classification or the detection of glaucomatous disc changes and RNFL defects. The three models were trained using images from three public data sets, namely Kaggle [17], MESSIDOR [73] and IDRiD [18]. Since these databases do not have labels for any of these tasks, a semi-supervised learning approach was followed. Models pre-trained on a private data set were used to assign labels to the images on each of the public sets. The same architectures were then trained from scratch on the combined data set to produce final predictions. The fi nal gl aucoma li kelihood wa s assigned by doing: $\max \{$ glaucomatous disc change, RNFL defect + glaucoma suspect $/ 2\}$.

\section{WinterFell}

1) OD/OC segmentation: The $\mathrm{ONH}$ was initially detected using a Faster R-CNN [79]. This area was cropped in all the images, and two image processing techniques were applied on the outputs. The first a pproach c onsisted of selecting a standard image and then normalize the remaining ones using it as a reference, on each color channel. The second image version was the inverted green channel of the original RGB cropped image. Finally, a ResU-Net [80] model was applied on the resulting images for $\mathrm{OD} / \mathrm{OC}$ segmentation.

2) Glaucoma classification: An ensemble of ResNets [41] (101 and 152) and DensNets [74] (169 and 201) was used for classification. The networks were pre-trained on ImageNet and separately fine-tuned using ORIGA, based on the loglikelihood loss. Each model was trained on cropped versions of the inputs images, centered in the $\mathrm{ONH}$ and on three different color space (RGB, HSV and the inverted green channel). Hence, $4 \times 3=12$ different models were produced. The final result was obtained by taking the mode of the binary decisions of each network. If the predicted label was glaucoma, the maximum confidence score was used as a final likelihood. On the contrary, if the image was labeled as non-glaucomatous, then the minimum score was applied.

\footnotetext{
${ }^{13}$ https://bitbucket.org/woalsdnd/refuge/src
} 